\title{
Magnetic Mn-Doped Ge Nanostructures
}

\author{
Faxian Xiu \\ Department of Electrical and Computer Engineering, Iowa State University, Ames, IA 50011, USA
}

Correspondence should be addressed to Faxian Xiu, faxian@iastate.edu

Received 4 December 2011; Accepted 9 January 2012

Academic Editors: A. Dinia, K. Haenen, E. Liarokapis, and C. Trallero-Giner

Copyright ( 2012 Faxian Xiu. This is an open access article distributed under the Creative Commons Attribution License, which permits unrestricted use, distribution, and reproduction in any medium, provided the original work is properly cited.

\begin{abstract}
With the seemly limit of scaling on CMOS microelectronics fast approaching, spintronics has received enormous attention as it promises next-generation nanometric magnetoelectronic devices; particularly, the electric field control of ferromagnetic transition in dilute magnetic semiconductor (DMS) systems offers the magnetoelectronic devices a potential for low power consumption and low variability. Special attention has been given to technologically important group IV semiconductor based DMSs, with a prominent position for Mn doped Ge. In this paper, we will first review the current theoretical understanding on the ferromagnetism in $\mathrm{Mn}_{x} \mathrm{Ge}_{1-x}$ DMS, pointing out the possible physics models underlying the complicated ferromagnetic behavior of $\mathrm{Mn}_{x} \mathrm{Ge}_{1-x}$. Then we carry out detailed analysis of $\mathrm{Mn}_{x} \mathrm{Ge}_{1-x}$ thin films and nanostructures grown by molecular beam epitaxy. We show that with zero and one dimension quantum structures, superior magnetic properties of $\mathrm{Mn}_{x} \mathrm{Ge}_{1-x}$ compared with bulk films can be obtained. More importantly, with $\mathrm{Mn}_{x} \mathrm{Ge}_{1-x}$ nanostructures, such as quantum dots, we demonstrate a field controlled ferromagnetism up to $100 \mathrm{~K}$. Finally we provide a prospective of the future development of ferromagnetic field effect transistors and magnetic tunneling junctions/memories using dilute and metallic $\mathrm{Mn}_{x} \mathrm{Ge}_{1-x}$ dots, respectively. We also point out the bottleneck problems in these fields and rendering possible solutions to realize practical spintronic devices.
\end{abstract}

\section{Introduction}

Dilute magnetic semiconductors (DMSs) attract tremendous interest as emerging candidates for the microelectronics industry due to their uniqueness in exhibiting spin-dependent magneto-electro-optical properties. Their distinctive material characteristics such as spin-dependent coupling between semiconductor bands and the localized states promises magnetoelectric effect-the modulation of magnetic properties by an applied electric field-in semiconductors $[1,2]$. Thus, a wide variety of semiconductor devices can be envisaged [3], such as spin polarized light-emitting diodes, lasers $[4,5]$ and spin transistor logic devices $[2,6,7]$. The development of these devices is considered as a possible route for extending the semiconductor scaling roadmap to spin-added electronics [8].

DMS materials can be developed by alloying semiconductors with several percentages of magnetic transition elements (with 3-d orbitals), such as $\mathrm{Fe}, \mathrm{Mn}, \mathrm{Co}, \mathrm{Ni}$, and $\mathrm{V}$ [9]. The anticipated outcome due to this doping is the fact that the transition elements occupying substitutional sites hybridize with the semiconductor host via the $s p$ - $d$ exchange interaction and help to enhance the spin-dependent transport, which in turn increase both the magnetization and the Curie temperature $\left(T_{c}\right)$ of the semiconductor system [10]. This means that the transition metal doping can generate strong spin-dependent coupling states in semiconductor systems, which may be further modulated by an electric field [2]. Since the band gap engineering and crystal structures of a DMS material are compatible with other semiconductors, they offer significant integration advantages as well [3].

The dependence of the transition temperature on the density of mobile carriers is considered as a good evidence for a genuine DMS. However, the key challenge for the growth of DMS materials is the ability to control synthesis of transition metals in semiconductors, aimed at increasing the doping concentration in order to realize room-temperature ferromagnetism. Thus, achieving both semiconducting and ferromagnetic property at room temperature has become a great challenge. Unfortunately, this is hampered by low solubility of transition metal species with a strong tendency to form metallic clusters or heterogeneous regions within 
the matrix. So the primary research on DMS growth is to produce samples with uniform doping and being free of metallic precipitates.

Substantial theoretical and experimental work were carried out in the past two decades, either to predict or to measure the magnetic properties on a variety of semiconductor materials, including binary and ternary compounds such as III-V, II-VI, IV-VI, VI oxides, III nitrides, and group IV systems. Among them, (Ga, Mn)As became the first and the mostly studied DMSs since 1996 [11]; but to our knowledge, some studies lacked the threshold doping profile to push its curie temperature to room temperature and above (currently around $190 \mathrm{~K}[12-15]$ ). Since then, substantial research has been carried out in III-V and II-VI semiconductors to improve the $T_{c}$ by increasing the solubility limit and meanwhile minimizing self-compensation effect of magnetic structures, using radical techniques such as delta doping of modulation-doped quantum structures [16-19]. Another approach to achieve high- $T_{c}$ DMS is to use transition metal-doped wide bandgap nitrides and oxides, such as $\mathrm{GaN}, \mathrm{AlN}, \mathrm{ZnO}$, and $\mathrm{TiO}_{2}$. In these materials, high magnetic impurity concentrations are needed due to the lack of longrange interaction among magnetic spins. However, there have been only limited reports on the carrier mediated effect in the wide band gap materials at room temperature [2022]. Though considerable amount of work has been done on III-V and II-VI DMSs $[2,23]$, the lack of high $T_{c}$ and the difficulty in achieving electric field modulated ferromagnetism (carrier-mediated ferromagnetism) at or above room temperature deters them from being considered for semiconductor integration.

Group IV semiconductors are of particular interest to the spintronics technology because of their enhanced spin lifetime and coherent length due to their low spin-orbit coupling and lattice inversion symmetry $[24,25]$. There is also considerable interest for transition metal-doped group IV semiconductors [26-36] to the semiconductor industry, owing in part to their excellent compatibility with silicon process technology. Several transition metals -including the use of single and codoping of elements such as $\mathrm{Mn}, \mathrm{Cr}, \mathrm{Co}, \mathrm{Fe}$, $\mathrm{Co}-\mathrm{Mn}$, and $\mathrm{Fe}-\mathrm{Mn}$ - have been used as magnetic dopants in both $\mathrm{Si}$ and Ge either through blanket implantation or through in situ doping during epitaxial growth process. Both these materials are reported to be ferromagnetic $[33,37,38]$. The reported experimental results also clearly showed the presence of carrier-mediated ferromagnetism in DMS Ge $[39,40]$. A recent study predicted that the $T_{c}$ could be increased by enhancing the substitutional doping of $\mathrm{Mn}$ in $\mathrm{Ge}$ and Si, via codoping - by adding conventional electronic dopants such as As or P during the Mn doping process [41]. Theories [42] and experiments [29, 43] further suggest that the ferromagnetic transition temperature is related to the ratio of interstitial to substitutional Mn similar to that in the group III-V system [10].

Considering the fact that, nanosystems such as nanowires and quantum dots (QDs) are the versatile building blocks for both fundamental studies in nanoscale and the assembly of present day functional devices, low-dimensional Ge DMS systems such as $\mathrm{Mn}_{x} \mathrm{Ge}_{1-x}$ nanowires $[13,30,44,45]$ and
QDs [46] have also been reported to have room temperature ferromagnetism. The above results are supported by modeling results [47] although carrier-mediated exchange has not yet to be experimentally verified in nanowires. Similarly, high Curie temperatures have been observed in other transition metal-doped semiconductor nanostructures such as II-VI (including $\mathrm{ZnO}[48-50], \mathrm{ZnS}[51,52]$, CdS [52]), and III-V (including GaN [52, 53], GaAs [54]), but only inconclusive reports have been published on the magnetoelectric transport in these nanostructures. It could be attributed to the complicated ferromagnetic properties caused by precipitates, second-phase alloys and nanometerscale clusters. Therefore, it is important and indispensable to differentiate their contributions. Unfortunately, there is still a lack of fundamental understanding of the growth process and spin-dependent states in these systems that gives an impetus to extensive investigations into these and related material systems.

In this paper, we will first introduce the state-of-art theoretical understanding of ferromagnetism in group IV DMSs, particularly pointing out the possible physics models underlying the complicated ferromagnetic behavior of MnGe. Then, we primarily focus on the magnetic characterizations of epitaxially grown $\mathrm{Mn}_{x} \mathrm{Ge}_{1-x}$ thin films ranging from three-dimensional thin films to zero-dimensional QDs. It is found that when the material dimension decreases (to zero), it evidences a change of material structures being free of precipitates and a significant increase of $T_{c}$ over $400 \mathrm{~K}$. The preliminary explanation on these behaviors can be attributed to the carrier confinement in the DMS nanostructures which strengthens hole localization and subsequently enhances the thermal stability of magnetic polarons, thus giving rise to a higher $T_{c}$ than those of bulk films.

Another important aspect of DMS research lies in the electric-field-controlled ferromagnetism. We emphasize the existing experimental efforts and achievement in this direction and provide a detailed description on the field-controlled ferromagnetism of the $\mathrm{Mn}_{x} \mathrm{Ge}_{1-x}$ nanostructures. We show that the ferromagnetism for the $\mathrm{Mn}_{x} \mathrm{Ge}_{1-x}$ nanostructures, such as QDs, can be manipulated via the control of gate voltage in a gate metal-oxide-semiconductor (MOS) structure (up to $100 \mathrm{~K}$ ). These experimental data suggest a hole-mediated ferromagnetism as controlled by a gate bias and promise a potential of using $\mathrm{Mn}_{x} \mathrm{Ge}_{1-x}$ nanostructures to achieve spintronic devices. Beyond the scope of DMSs, we will present the metallic $\mathrm{Mn}_{x} \mathrm{Ge}_{1-x}$ with $x \sim 20 \%$, in which a constant Curie temperature was observed. The emphasis, however, is the manipulation of these metallic dots into selfassembled periodic arrays for potential device applications. Finally, we render general comments on the development of the $\mathrm{Mn}_{x} \mathrm{Ge}_{1-x}$ system and possible research schemes to develop spintronics devices based on this material system.

\section{Theories of Ferromagnetism in Group IV DMS}

As in other popular dilute magnetic semiconductors such as ( $\mathrm{Ga}, \mathrm{Mn}) \mathrm{As}$, the formation of magnetic order and the origin 
of ferromagnetism in $\mathrm{Mn}_{x} \mathrm{Ge}_{1-x}$ can be described (within the parametric limits) either using complementary mean field approximation models such as the Zener-Kinetic exchange [55], the Ruderman-Kittel-Kasuya-Yoshida (RKKY) [56] interaction, and percolation theory [57] or using density functional theory such as $a b$ initio full-potential augmented plane wave (FLAPW) [58] electronic calculations. Interestingly, the Zener-kinetic exchange model proposed in 1950 could be used for interpreting many of the experimental results in transition metal-doped semiconductors [59-61] besides the transition metals themselves. All the above theoretical approaches have tried to address and to a certain extent successfully answered chemical, electronic, and magnetic properties of the transition metal-doped group IV semiconductors within the context of (1) the nature of transition metal impurities, (2) the origin of ferromagnetism, (3) the influence of dopants on the $T_{c},(4)$ the solubility limits of carrier density on the chemical nature, (5) the influence of carrier density on the electronic structure, and (6) the influence of different hosts on the electronic structure. Within the above theoretical approaches, these models assume that the ferromagnetism in transition metal-doped Ge system is mediated through exchange coupling between the carriers and the interacting lattice. This means that, the interaction is through $p$ - $d$ orbital hybridization between the $d$ levels and the valence band $p$ states of Ge. According to these models, as the number of dopant carriers increases both the electronic mobility and the ferromagnetic ordering of the system also increases along with the $T_{c}$. Thus, the assumption is that the strong exchange coupling between the $p$ and $d$ orbitals leads to strong kinetic exchange coupling between the spinpolarized holes and spin polarized TM substitutional vacancies. Among all the 3- $d$ TM impurities, $[\mathrm{TM}=\mathrm{V}, \mathrm{Cr}, \mathrm{Mn}$, $\mathrm{Fe}, \mathrm{Co}, \mathrm{Ni}], \mathrm{Mn}$ is the most favored dopant. This is because compared to other dopants, Mn favors less clustering, low nonuniform doping distribution and higher substitutional doping over interstitials. The $a b$ initio electronic structure calculations within density functional theory show that such doping behaviors could result in relatively higher carrier hole concentrations and increased magnetic moments [27, 58]. Complementary theoretical work using the frozen-magnon scheme also shows that the ferromagnetism is produced only through holes and $\mathrm{Mn}$ is a good source for generating holes in Ge system [62].

In addition, some of the other factors that determine the ferromagnetic strengths in Ge are (1) disorder of $\mathrm{Mn}$ site locations, (2) distance between Mn-Mn atoms, (3) solid solubility limits and preferential surface orientation for interstitial and substitution site formation [63-65], and (4) codoping of Co or Cr with Mn. (They were found to reduce cluster formation but not to significantly contribute to the ferromagnetism in the system $[66,67]$.) According to full potential linearized augmented plane wave (FLAPW) calculations made on the RKKY model, the coupling between the Mn atoms could be ferromagnetic (FM) or antiferromagnetic (AFM) in nature but the interaction is very localized to Mn sites and depends on the distance between Mn atoms: the density of states decreases rapidly as the Ge atom moves away from Mn [58]. However, disorder of Mn site locations is found to influence the ferromagnetic strength and the transition temperature. In addition, the impact of inherent disorder is also confirmed both by experiments and by the mean field approximation $[57,68,69]$. Some of the above models found themselves hard to explain the long-range exchange interaction and coupling strength when there is varying dopant concentration and disorder ranging from high resistive state to half metallic state. On the other hand, there are attempts to find solutions for disorder-dependent magnetic variation using Percolation theory-based mean field approximation calculation $[57,70]$. Percolation theory provides possible explanations for the experimentally observed low values of saturation magnetization in low carrier density $\mathrm{Mn}_{x} \mathrm{Ge}_{1-x}$ because the infinite cluster of percolating bound magnetic polarons triggering the long-range ferromagnetic order leaves out a large number of $\mathrm{Mn}$ moments at temperatures less than $T_{c}$. However, the role of disorder with respect to insulating and conductive metallic phases and their effects on mobility is still not well understood. Conversely, the FLAPW calculations using the density functional theory shows that Mn favors long-range ferromagnetic alignment over shortrange antiferromagnetic alignment and this increases with the increase of $\mathrm{Mn}$ content which is in agreement with many of the experimental results [58]. The estimated Curie temperature is within the range of $134-400 \mathrm{~K}$ and is in agreement with experimental and theoretical results [47].

However, there are also conflicting reports that longrange FM order could only happen at low temperature (12 K) and that at low temperature MnGe could show only spin glass behavior due to the intercluster formation between the FM Mn-rich clusters [42, 71]. With these reports, it is understood that the magnetic phases are more favored over paramagnetic phases by an energy difference of $200 \mathrm{meV} / \mathrm{Mn}$ atom [58]. The report also states that the $\mathrm{Mn}_{x} \mathrm{Ge}_{1-x}$ system is very close to half metallicity irrespective of the amount of doping concentration $[58,72]$. Electronic band structure calculations using density of states (DOSs) show that the binding energy of the Ge $4 s$ states are basically unaffected by the exchange splitting of $\mathrm{Mn} d$ states and the valence band maximum of the minority spin channel in $\mathrm{Mn}_{x} \mathrm{Ge}_{1-x}$ reaches $E_{F}$ at Gamma $(T)$ such that the spin gap becomes zero $[58,72]$. In other words, the DOS shows a valley around $E_{F}$ and is strictly zero at $E_{F}$ in the minority spin channel. On the other hand, the energy gap is indirect and the bands around $E_{F}$ in the majority spin channel arise from $\mathrm{Mn} d$ and Ge $p$ hybridizations so as to give rise to hole pockets closer to the gamma point with a localized magnetic moment of about $3 \mu_{B}$. This could also be explained using chemical and structural configurations of $\mathrm{Mn}$ in Ge. The different chemical species determine the relative positions of the anion and cation atomic energy levels in the $\mathrm{Mn}_{x} \mathrm{Ge}_{1-x}$ while the different anion size dictates essentially the equilibrium lattice constants [58]. This essentially explains the carrier-mediated effect in $\mathrm{Mn}_{x} \mathrm{Ge}_{1-x}$ even though the system is closer to half metallic. The aim of all these theoretical works is to build foundation for achieving carrier-mediated ferromagnetism at temperatures above room temperature. It is understood that this can be achieved only by growing cluster-free and uniformly doped $\mathrm{Mn}_{x} \mathrm{Ge}_{1-x}$ samples. Experimental reports 
show that $T_{c}$ as high as $400 \mathrm{~K}$ is obtained for cluster-free $\mathrm{Mn}_{x} \mathrm{Ge}_{1-x}$ samples grown (1) using subsurfactant epitaxial method [73], (2) epitaxial QDs [40], and (3) nanowire structures $[13,30,44]$. However, there are only very few theoretical studies depicting the relation between the quantum confinement and its influence on the origin of ferromagnetism in low-dimensional DMS structures [74-76]. It apparently lacks of systematic theoretical study particularly on $\mathrm{Mn}_{x} \mathrm{Ge}_{1-x}$ QDs. Therefore, it will be interesting to understand the ability of the quantum confinement phenomenon to retain spin polarization in $\mathrm{Mn}_{x} \mathrm{Ge}_{1-x}$ QDs structure. Studies on III-V QDs using LSDA approximation show that, unlike in the bulk structures, adding a single or multiple carriers in a magnetic QD can both strongly change the total carrier spin and the temperature of the onset of magnetization and this can be modulated by modifying the quantum confinement and the strength of coulomb interactions. These theoretical results must have ramification in the high Curie temperature $\mathrm{Mn}_{x} \mathrm{Ge}_{1-x}$ QDs as well since our studies also reveal similar characteristics. But, all of the effects including high $T_{c}$ that we observe cannot be explained without invoking tiny defects either at the interface or on the surface although the conventional transmission electron microscopy (TEM) cannot reveal their existence due to the limited resolution. All of the above material and electronic properties have been studied in order to understand how feasible a DMS material is for carrier-mediated ferromagnetism.

\section{Growth and Characterizations of Group IV DMS and Nanostructures}

3.1. Overview of the Mn-Doped Ge. As mentioned above, the Ge-based DMS has attracted extensive attention due to its possibility to be integrated with the mainstream Si microelectronics, in which they may be used to enhance the functionality of Si-integrated circuits [27]. In particular, the holemediated effect discovered in $\mathrm{Mn}_{x} \mathrm{Ge}_{1-x}$ DMS opens up tremendous possibilities to realize spintronic devices with advantages in reducing power dissipation and increasing new functionalities, leading to perhaps normally off computers. To date, there are many reports on the $\mathrm{Mn}_{x} \mathrm{Ge}_{1-x}$ growth and characterizations by molecular beam epitaxy (MBE) [27, 28, $31,33,43,77-85]$, ion implantation [86-93], and bulk crystal growth [37, 94]. We can divide them here, for an easier orientation, into two groups: those that cover fundamental studies of phase formation, ferromagnetism, and transport properties $[26,27,29,31,33,35-37,43-45,64,77-85$, 94$115]$, and those that aim to minimize cluster formation and enhance Curie temperatures via codoping methods $[28,32$, 34, 116-118].

Experimental results show that the Mn-doping process in $\mathrm{Mn}_{x} \mathrm{Ge}_{1-x}$ is complex. Both the $T_{c}$ and the saturation magnetization depend on the interplay of a variety of factors, which are ultimately determined by growth conditions and postannealing process [33]. The concentration and distribution of Mn dopants, the carrier density, the presence of common defects such as Mn interstitials and Mn clusters significantly influence the magnitude and interactions of the magnetic coupling [94]. In early 2002, Park et al.
[27] demonstrated ferromagnetic $\mathrm{Mn}_{x} \mathrm{Ge}_{1-x}$ thin films by low-temperature MBE. It was found that the $\mathrm{Mn}_{x} \mathrm{Ge}_{1-x}$ films were $p$-type semiconductors in character with hole concentration of $10^{19} \sim 10^{20} \mathrm{~cm}^{-3}$ and exhibited pronounced extraordinary Hall effect. The $T_{c}$ of the $\mathrm{Mn}_{x} \mathrm{Ge}_{1-x}$ thin films increased linearly with Mn concentration from 25 to $116 \mathrm{~K}$ $(\mathrm{Mn} \leq 3.5 \%)$. Field-controlled ferromagnetism was also observed in a simple gated structure through application of a gate voltage $( \pm 0.5 \mathrm{~V})$, showing a clear hole-mediated effect at $50 \mathrm{~K}$. Such a small gate voltage presents an excellent comparability with conventional low voltage circuitry. The origin of ferromagnetic order was understood in the frame of localspin density approximation (LSDA), where strong hybridization between Mn $d$ states of $\mathrm{T}_{2}$ symmetry with Ge $p$ states leads to the configuration $\mathrm{e}(\uparrow)^{2} \mathrm{~T}_{2}(\uparrow)^{2} \mathrm{~T}_{2}(\downarrow)^{1}$, with a magnetic moment of $3 \mu_{B}$. However, the LSDA calculations gave overestimated $T_{c}$ which was attributed to the incomplete activation of $\mathrm{Mn}$ in experiments and the absence of hole compensation in the simulation [27].

Since then, various preparation techniques were employed in order to produce Mn-doped Ge DMS, including aforementioned MBE [28, 31, 33, 43, 77-85], single-crystal growth $[37,94]$, and ion implantation [86-93], aiming to further increase $T_{c}$ and to obtain the electric field controllability at room temperature [94]. Among these efforts, Cho et al. [37] reported the synthesis of Mn-doped bulk Ge single crystals with $6 \%$ of $\mathrm{Mn}$ and a high ferromagnetic order at about $285 \mathrm{~K}$ via a vertical gradient solidification method. The origin of the high $T_{c}$ was found to be complex because of the presence of dilute and dense $\mathrm{Mn}$-doped regions. Jaeger et al. [77], however, pointed out that the magnetic properties of these samples were clearly dominated by the presence of the intermetallic compound $\mathrm{Mn}_{11} \mathrm{Ge}_{8}$. This was indeed observed by Biegger et al. [94], where similar $\mathrm{Mn}_{x} \mathrm{Ge}_{1-x}$ crystals were produced via Bridgman's crystal growth technique and intermetallic compounds were found in both Mn-rich and Mn-poor regions. These experiments suggest that the bulk $\mathrm{Mn}_{x} \mathrm{Ge}_{1-x}$ crystals produced at over $1000^{\circ} \mathrm{C}$ may not be an appropriate method for the $\mathrm{Mn}_{x} \mathrm{Ge}_{1-x}$ DMS preparation [94].

Apart from the bulk crystal growth, MBE has been widely recognized as a power tool for DMS growth since it can provide nonequilibrium conditions to enhance the incorporation of Mn dopant [27]. Via this technique, Mn doping was found to be extremely sensitive to growth conditions, particularly to the growth temperature. Excellent work on this aspect can be found in references [32, 33, 43, 79, 81, 83, 85]. In general, at low growth temperatures $\left(T_{b}<120^{\circ} \mathrm{C}\right)$, the diffusion of $\mathrm{Mn}$ atoms leads to the formation of $\mathrm{Mn}$-rich nanostructures, such as nanocolumns [82] and nanodots [81] with irregular shapes. These nanostructures could contain a high Mn concentration up to $38 \% 33$, while the surrounding matrix remains a low $\mathrm{Mn}$ concentration less than $1 \%$. A perfect lattice coherence was found in the proximity of the nanostructures, indicative of a strong compression for coherent nanostructure and a large tension for the surrounding matrix [82]. These observations are in good agreement with theoretical predictions of a spinodal decomposition [119], which occurs under a layer-by-layer growth mode with 
TABLE 1: A brief summary of the MnGe growth and characterizations.

\begin{tabular}{|c|c|c|c|c|c|c|}
\hline Mn-doped Ge & Growth method & Mn (\%) & $T_{c}(\mathrm{~K})$ & Origin of FM & Field controlled FM & Ref. \\
\hline Thin films & $\mathrm{MBE}$ & $0.6 \sim 3.5$ & $25 \sim 116$ & DMS & $50 \mathrm{~K}$ & {$[27]$} \\
\hline Bulk single crystal & Sintering & 6.2 & $150 \sim 285$ & $\mathrm{Mn}_{5} \mathrm{Ge}_{3}, \mathrm{Mn}_{11} \mathrm{Ge}_{8}$ & No & {$[37,94]$} \\
\hline Thin films & MBE & $<6$ & $\sim 300$ & $\mathrm{Mn}_{5} \mathrm{Ge}_{3}$ & No & {$[36,77,98]$} \\
\hline Thin films (with $\mathrm{MnGe}_{2}$ ) & $\mathrm{MBE}$ & 6 & $>400$ & $\mathrm{MnGe}_{2}$ clusters & No & {$[33]$} \\
\hline Thin films & Implantation & A few & $\sim 300$ & $\mathrm{Mn}_{5} \mathrm{Ge}_{3}, \mathrm{Mn}_{11} \mathrm{Ge}_{8}$ & No & {$[39,88,89,92]$} \\
\hline
\end{tabular}

strong pairing attraction between $\mathrm{Mn}$ atoms and a tendency of surface diffusion of $\mathrm{Mn}$ atoms. These theoretical calculations successfully explained the formation of nanostructures in $(\mathrm{Ga}, \mathrm{Mn}) \mathrm{N}$ and $(\mathrm{Zn}, \mathrm{Cr}) \mathrm{Te})[82,119]$, which can be also readily applied to the $\mathrm{Mn}_{x} \mathrm{Ge}_{1-x}$ system [82]. The magnetic properties, however, exhibit various characteristics depending on the nanostructure size and the Mn concentration. In most cases, zero-field-cooled (ZFC) and field-cooled (FC) magnetizations show superferromagnetic properties with a blocking temperature of $\sim 15 \mathrm{~K}$. It is now clear that such a low blocking temperature is originated from the coherent nanostructures with a diameter below several nanometers. Curie temperatures of these nanostructures were also identified to be below $170 \mathrm{~K}$ and varied with different Mn concentration. No $\mathrm{Mn}_{5} \mathrm{Ge}_{3}$ metallic phases were observed at low temperature regime $\left(T_{b}<120^{\circ} \mathrm{C}\right)$.

Bougeard et al. [81] also found that when the dimension of the nanostructures further reduced, the films showed no overall spontaneous magnetization down to $2 \mathrm{~K}$. TEM results were interpreted in terms of an assembly of superparamagnetic moments developing in the dense distribution of nanometer-sized clusters. In addition to the single crystalline $\mathrm{Mn}_{x} \mathrm{Ge}_{1-x}$, homogeneous nanoclusters were also observed under certain growth conditions and contributed to ferromagnetic order below $100 \mathrm{~K}[82,107]$. To render a general understanding of the $\mathrm{Mn}_{x} \mathrm{Ge}_{1-x}$ magnetic properties, Jaeger et al. [77] presented a detailed study on $\mathrm{Mn}_{0.04} \mathrm{Ge}_{0.96}$ and $\mathrm{Mn}_{0.20} \mathrm{Ge}_{0.80}$ thin films grown at low temperatures and related their magnetic behavior to spin-glass. A frozen magnetic state at low temperatures was observed and attributed to the formation of nanostructures. This is in a good agreement with Devillers et al. [82]. Therefore, it can be concluded that the $\mathrm{Mn}_{x} \mathrm{Ge}_{1-x}$ magnetic semiconductor is not a conventional ferromagnet since its magnetic properties are significantly complicated by the formation of nanostructures and metallic clusters.

When the growth temperature falls into $120 \sim 145^{\circ} \mathrm{C}$, the $\mathrm{Mn}_{5} \mathrm{Ge}_{3}$ nanoclusters start to develop and dominate the magnetic property with a Curie temperature of $296 \mathrm{~K}$ [33, 82]. This phase, frequently observed at high-temperature growth, is the most stable (Mn, Ge) alloy. The other stable compound $\mathrm{Mn}_{11} \mathrm{Ge}_{8}$ was also observed in nanocrystallites surrounded with pure Ge [83]. Although they are ferromagnetic, the metallic character considerably jeopardizes their potential for spintronic applications. Under a narrow growth window around $130^{\circ} \mathrm{C}$, another phase $\mathrm{MnGe}_{2}$ nanocolumns could be developed with a $\sim 33 \%$ of $\mathrm{Mn}$ and a $T_{c}$ beyond $400 \mathrm{~K}$ [33]. A remarkable feature of these clusters is the large magnetoresistance due to geometrical effects. Further increasing the growth temperature leads to the dominating magnetic behavior from the $\mathrm{Mn}_{5} \mathrm{Ge}_{3}$ clusters. As the coherent nanostructures grow larger at high temperature growth, higher blocking temperatures $(\sim 30 \mathrm{~K})$ were also obtained [82].

To minimize the phase separation, Mn codoping with Co was attempted to stabilize structures at high Mn-doping concentration $[28,34,116]$. It showed that codoping with Co can dramatically reduce phase separation and diffusion of Mn within the Ge lattice while it magnetically complements $\mathrm{Mn}$. The measured strain states indicate the critical role played by substitutional Co with its strong tendency to dimerize with interstitial $\mathrm{Mn}$ [34]. The highest $T_{c}$ achieved so far is about $270 \mathrm{~K}$ [28]. Similarly, Gareev et al. [118] reported on the codoping of Fe to achieve a $T_{c}$ of $209 \mathrm{~K}$. Recent polarized neutron reflectivity measurements provided evidence to show no segregation and lower clustering tendencies for higher Fe doping [117], which further supported codoping approach in $\mathrm{Mn}_{x} \mathrm{Ge}_{1-x}$.

Ion implantation was also attempted due to its efficiency of $\mathrm{Mn}$ incorporation [92]. However, extensive experiments revealed that $\mathrm{Mn}$-rich precipitates were developed and buried in a crystalline Ge matrix [86-93] in a much similar manner to that of the thin films grown by MBE $[32,43,81$, 83].

Although there has been much progress in producing high-quality $\mathrm{Mn}_{x} \mathrm{Ge}_{1-x}$ thin films, the formation of uncontrollable metallic clusters yet remains a challenge. High $T_{c}$ DMS thin films without any precipitations seem to be a critical obstacle for the fabrications of practical spintronic devices functioning at room temperature, not to mention the room-temperature-controlled ferromagnetism. Table 1 provides a brief summary of the current status of $\mathrm{Mn}_{x} \mathrm{Ge}_{1-x}$ on the growth method, the $T_{c}$, and the field controllability of ferromagnetism.

3.2. $M n_{x} G e_{1-x}$ by Molecular Beam Epitaxy. To gain further insight into the complex growth of this material system, we have performed detailed structural and magnetic characterizations of $\mathrm{Mn}_{x} \mathrm{Ge}_{1-x}$ grown by MBE with different thickness. Our experiments showed that the Mn-doping behavior and magnetic properties are dramatically different when the material dimension becomes smaller. Bulk $\mathrm{Mn}_{x} \mathrm{Ge}_{1-x}$ films always tend to form clusters no matter what growth conditions are. When it comes to one and zero dimensions, however, the cluster formation may be possibly limited because 


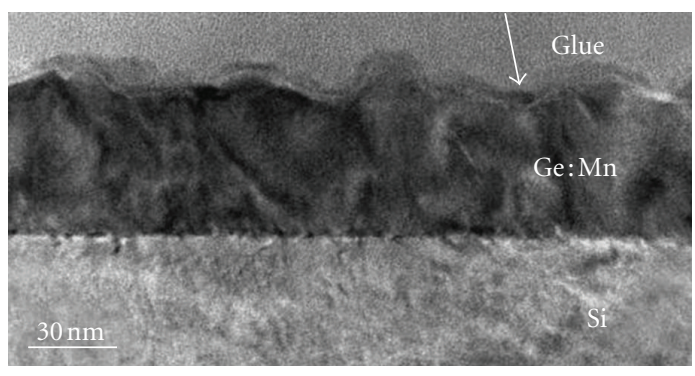

(a)

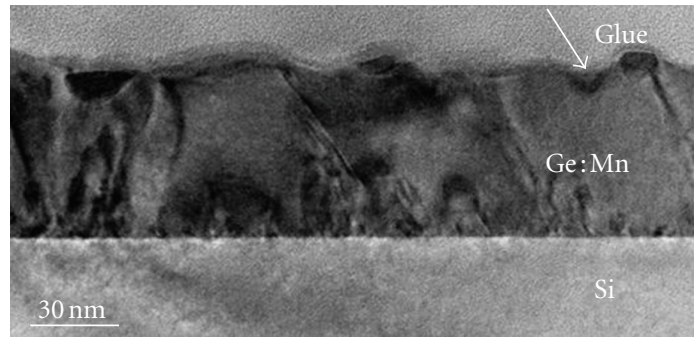

(b)

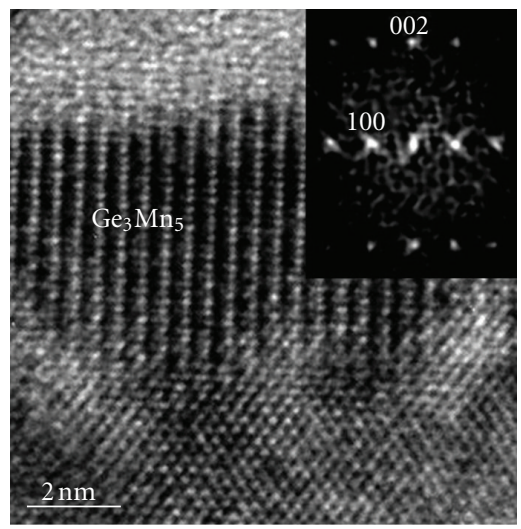

(e)

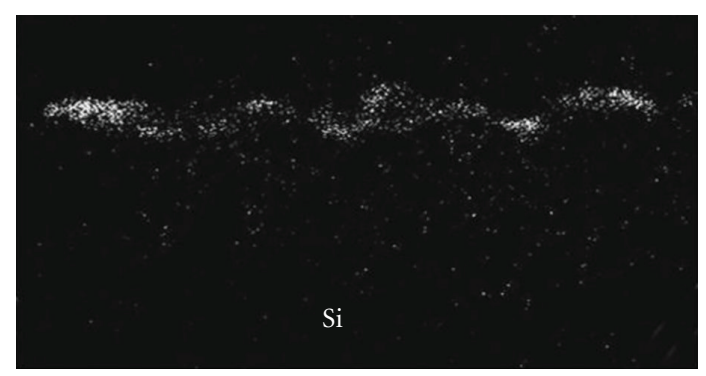

(c)

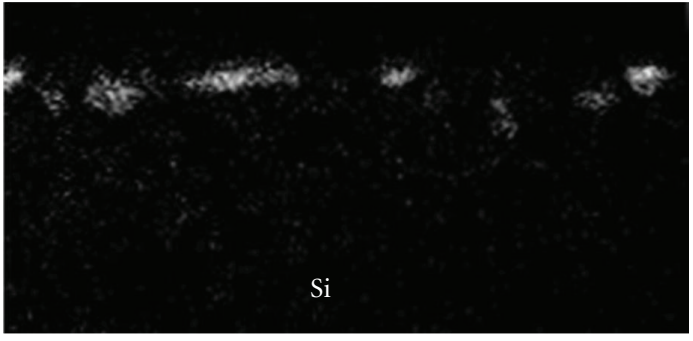

(d)

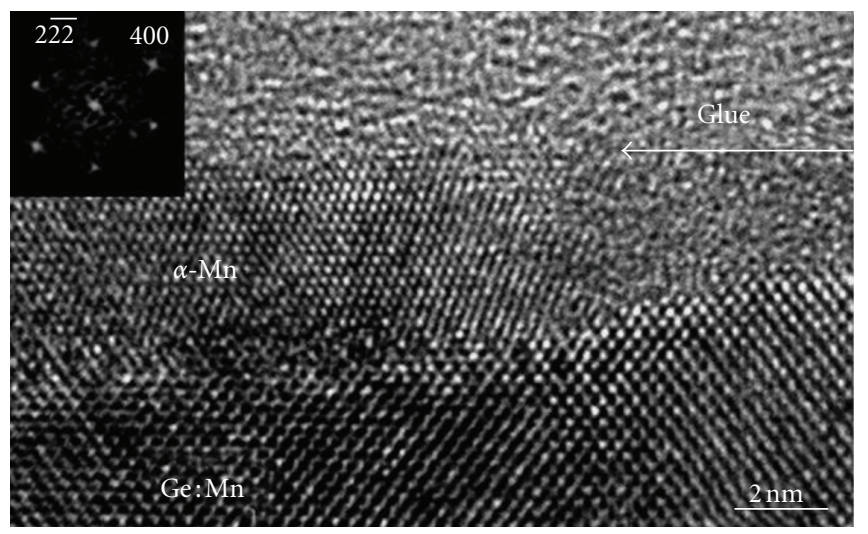

(f)

Figure 1: (a) As-grown sample at $250^{\circ} \mathrm{C}$, (b) annealed sample at $400^{\circ} \mathrm{C}$; (c) and (d) are corresponding Mn maps of (a) and (b), respectively; (e) HRTEM image of (a) typical Mn5Ge3 cluster; (f) HRTEM image of a typical $\alpha$-Mn metallic cluster epitaxially grown on the (Mn)Ge lattice. The insets in (e) and (f) are the corresponding FFT maps of the images.

the Mn concentration cannot accumulate enough and the strain can be easily accommodated to minimize the metallic clusters $[84,93]$. We further show that self-assembled $\mathrm{Mn}_{x} \mathrm{Ge}_{1-x}$ DMS QDs can be successfully grown on Si substrate with a high $T_{c}$ in excess of $400 \mathrm{~K}$. Finally, the electricalfield-controlled ferromagnetism was also demonstrated in MOS structures using these QDs as the channel layers, as elaborated in Section 4.

3.2.1. $M n_{x} G e_{1-x}$ Thin and Thick Films. Mn-doped Ge films were grown on Si substrates by a Perkin-Elmer solid source MBE. Si substrates were cleaned by $\mathrm{H}_{2} \mathrm{SO}_{4}: \mathrm{H}_{2} \mathrm{O}_{2}(5: 3)$ and $10 \% \mathrm{HF}$ with a final step of $\mathrm{HF}$ etching. The native oxide was removed by a $10 \mathrm{~min}$ flash at $800^{\circ} \mathrm{C}$ in the vacuum chamber. After that, a direct growth of $80 \mathrm{~nm}$ thick $\mathrm{Mn}_{x} \mathrm{Ge}_{1-x}$ on Si was carried out at $250^{\circ} \mathrm{C}$. A $5 \mathrm{~nm}$ Ge capping layer was deposited on the surface in order to prevent $\mathrm{Mn}_{x} \mathrm{Ge}_{1-x}$ from oxidizing. To probe the annealing effect, identical $\mathrm{Mn}_{x} \mathrm{Ge}_{1-x}$ thin films were grown repeatedly and followed by in situ annealing process at $400^{\circ} \mathrm{C}$ for $30 \mathrm{~min}$. All as-grown and annealed samples were characterized by high-resolution TEM (HR-TEM, FEI Tecnai F30 operating at $200 \mathrm{keV}$ ) and superconducting quantum interference device (SQUID, Quantum Design MPMS XL 5). Cross-section HR-TEM specimens along $\langle 110\rangle$ were prepared by using a tripod technique, followed by a final thinning using a Gatan precision ion polishing system.

Figures 1(a) and 1(b) show cross-sectional TEM images of the as-grown and annealed $80 \mathrm{~nm}$ thick $\mathrm{Mn}_{0.04} \mathrm{Ge}_{0.96}$. Nonuniform and discontinued rough films were observed in both cases. Figures 1(c) and 1(d) are their corresponding Mn elemental maps, respectively. $\mathrm{Mn}$ is seen to concentrate on top for both samples. Mn-containing clusters are confirmed near the top of the crystalline layer as indicated by the white arrow in Figure 1(a). A HR-TEM image clearly indicates that a Mn-containing cluster is formed on top of the Ge lattice as shown in Figure 1(e). Using the lattice constant of Ge 


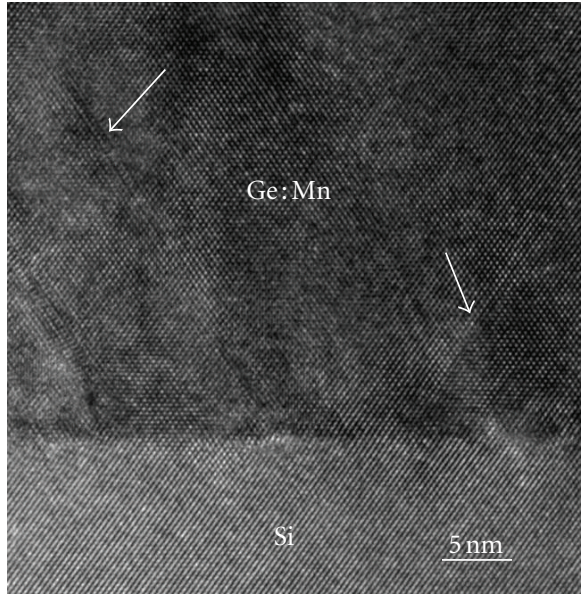

(a)

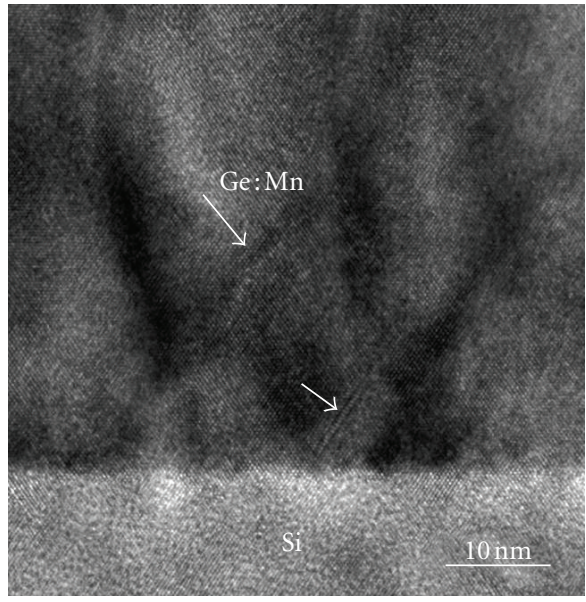

(b)

Figure 2: $80 \mathrm{~nm}$ thick Mn-doped Ge grown on $\mathrm{Si}$ at $250^{\circ} \mathrm{C}$, (a) as-grown film and (b) after $400^{\circ} \mathrm{C}$ annealing at for $30 \mathrm{~min}$. Stacking faults are marked by arrows.

as the reference, the lattice spacings of the Mn-containing cluster can be determined as 0.62 and $0.25 \mathrm{~nm}$, which are matched with the distances of (100) and (002) atomic planes of the hexagonal $\mathrm{Mn}_{5} \mathrm{Ge}_{3}$ phase. Fast Fourier transformation (FFT) map shown in the inset of Figure 1(e) also indicates that the cluster belongs to the hexagonal $\mathrm{Mn}_{5} \mathrm{Ge}_{3}$ phase. $\mathrm{Mn}_{5} \mathrm{Ge}_{3}$ clusters may be formed when the Mn concentration is sufficiently high for the nucleation of clusters [84].

Figure 2 shows the TEM cross-section of as-grown and $400^{\circ} \mathrm{C}$ annealed samples. Stacking faults along $\{111\}$ atomic planes are clearly observed in both cases. The annealing process does not dramatically affect the stacking-faults. However, the magnetic properties have a noticeable change. Figures 3(a) and 3(d) show clear hysteresis loops at $298 \mathrm{~K}$ for both the as-grown and annealed samples. Before annealing, the film shows a clear hysteresis at $10 \mathrm{~K}$ while a very small magnetic moment is observed at room temperature. After annealing, the hysteresis showed a significant change and the coercive field at $10 \mathrm{~K}$ decreased from 430 Oe to 92 Oe. For the as-grown film, $\mathrm{Mn}_{5} \mathrm{Ge}_{3}$ formed on the surface gave the $T_{c}$ of around $298 \mathrm{~K}$ as shown in Figure $3(\mathrm{~b})$. The blocking temperature was estimated to be about $250 \mathrm{~K}$. After annealing, both $\mathrm{Mn}_{5} \mathrm{Ge}_{3}$ and $\mathrm{Mn}$ clusters led to a complex magnetic behavior, showing multiple phase transitions at different temperatures in Figure 3(e). The $T_{c}$ increased from 298 to over $400 \mathrm{~K}$. Further investigation is needed to understand the change of the magnetic property. The Arrott's plots were made to confirm the $T_{c}$ in Figures 3(c) and 3(f). The estimated Curie temperatures for the as-grown and annealed samples were obtained to be $298 \mathrm{~K}$ and above $400 \mathrm{~K}$, respectively.

To understand the magnetic behavior of $\mathrm{Mn}$ in an ultrathin Ge, a $\mathrm{Mn}_{x} \mathrm{Ge}_{1-x}$ film with a thickness of about $15 \mathrm{~nm}$ was grown directly on Si substrate by following the same growth procedure as that for the $80 \mathrm{~nm}$ thick film. Figures 4(a) and 4(b) show typical cross-sectional TEM images and general morphologies of the as-grown and annealed samples. From these figures, a relative uniform crystalline layer, adjacent to the Si substrates topped with an amorphous layer, can be seen in both cases. The formation of amorphous layers is not clear yet, but it could be attributed to the growth conditions and the sample preparation for TEM when exposing to the air.

To understand the structural variation for the two cases, high-resolution TEM was carried out and typical $\langle 110\rangle$ zoneaxis HR-TEM images are shown in Figures 4(b) and 4(e) for the two cases, respectively, where the amorphous layers can be clearly seen. From these HR-TEM images, stacking faults in the crystalline layers can also be seen in both cases. No structural clusters were found through our extensive HRTEM investigations and no secondary phases were detected by our XRD measurements (not shown here). To further understand the $\mathrm{Mn}$ distribution in the $\mathrm{Mn}_{x} \mathrm{Ge}_{1-x}$ film (crystalline and amorphous), energy-filtered TEM was preformed. Figures 4(c) and 4(f) give the EF-TEM elemental maps of Mn that correspond to Figures 4(a) and 4(d), respectively. It is of interest to note that, in the as-grown case, a higher Mn concentration is found in the amorphous layer when comparing Figures 4(a) with 4(c). The overall concentration of $\mathrm{Mn}$ is low, so that the contrast in the Mn maps tends to be faint. Nevertheless, the distribution of Mn in the entire film can be clearly observed. In contrast, the Mn map (Figure 4(f)) in the annealed case shows some Mn distribution in the entire film, suggesting that the annealing has not only promoted crystallization, but also redistribute $\mathrm{Mn}$ in the entire $\mathrm{Mn}_{x} \mathrm{Ge}_{1-x}$ film. Nevertheless, a higher Mn concentration can still be observed in the surface region in the annealed sample (refer to Figure 4(f)). The fact that, for the as-grown sample, most of Mn was found in the topmost layer suggests that, during the growth of the $\mathrm{Mn}_{x} \mathrm{Ge}_{1-x}$ film, a dynamic Mn diffusion has taken place, even at a relatively low growth temperature of $250^{\circ} \mathrm{C}$ as in our case; $\mathrm{Mn}$ tends to diffuse towards the top surface.

Figure 5(a) shows the hysteresis loops at $298 \mathrm{~K}$ and $10 \mathrm{~K}$. A clear hysteresis at $298 \mathrm{~K}$ indicates room-temperature ferromagnetism. Figure 5(b) shows that the plot of the magnetic 

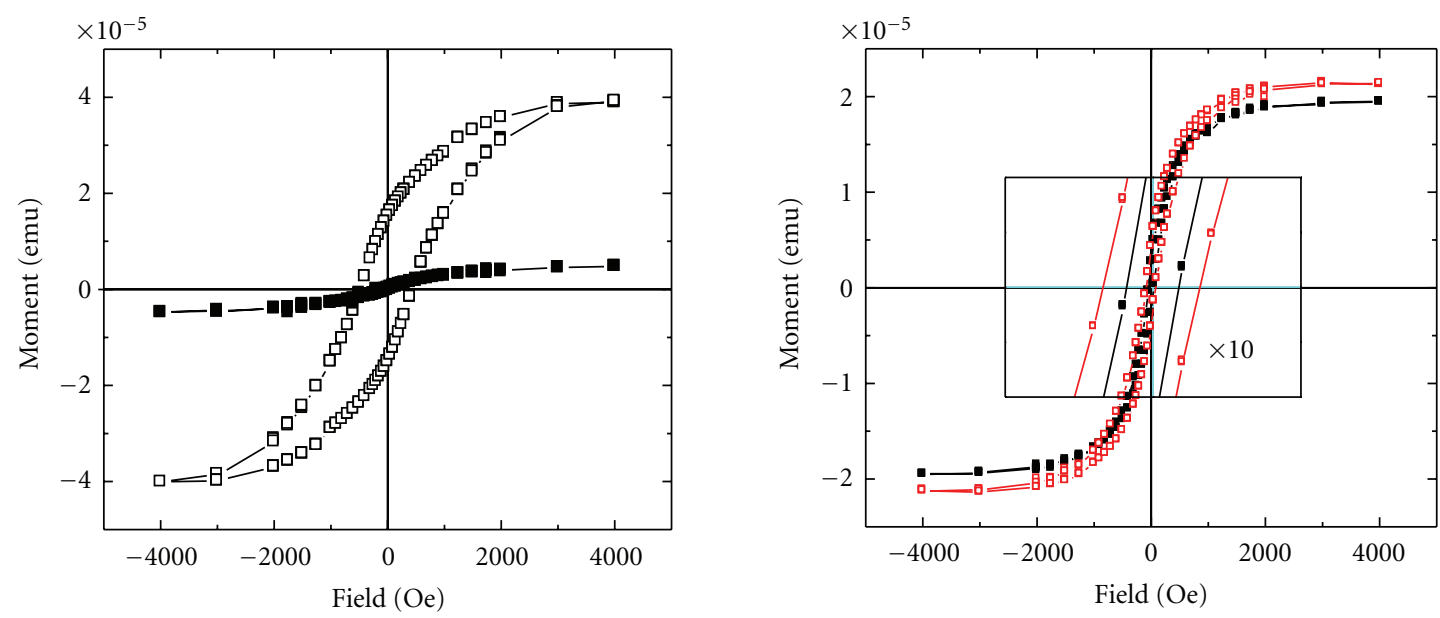

$$
\begin{aligned}
& \rightarrow-10 \mathrm{~K} \\
& \rightarrow-298 \mathrm{~K}
\end{aligned}
$$

$\times 10^{-5}$

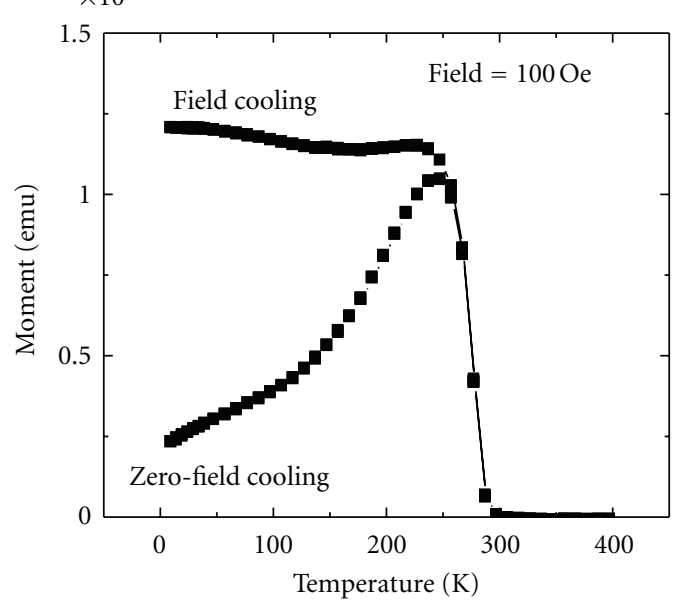

(b)

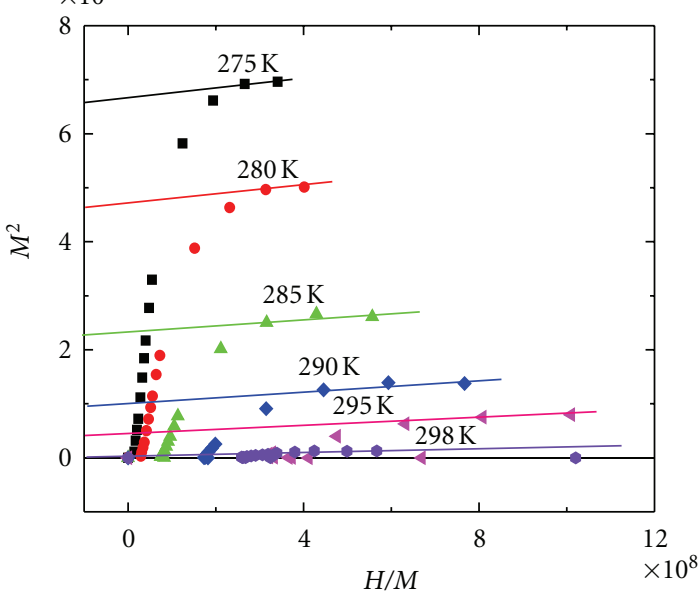

(c)

$$
\rightarrow-10 \mathrm{~K}
$$

$\rightarrow-298 \mathrm{~K}$

$$
\times 10^{-6} \quad \text { (d) }
$$

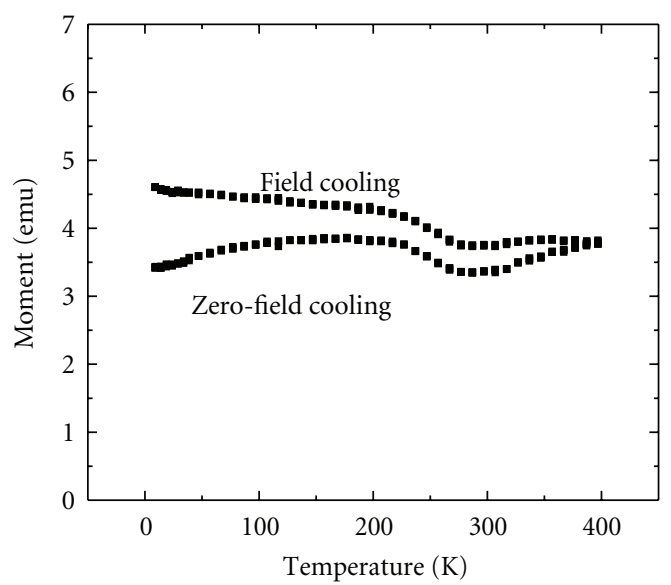

$\rightarrow$ Field $=100 \mathrm{Oe}$

$$
\times 10^{-10} \quad \text { (e) }
$$

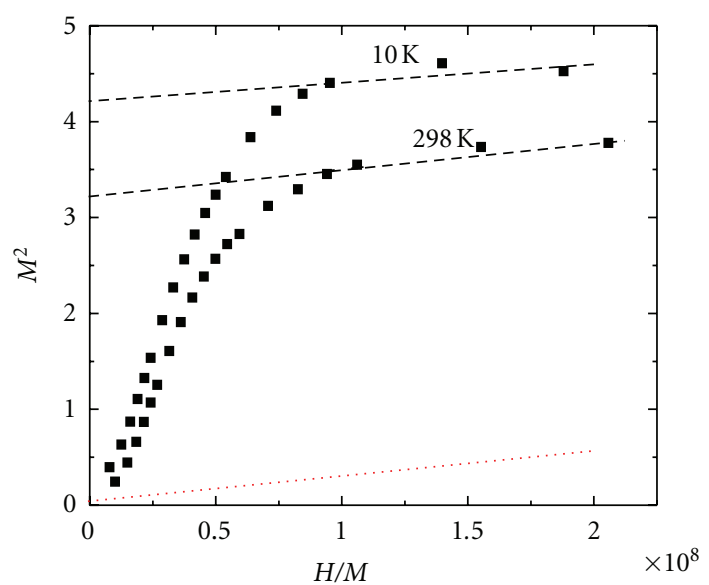

- Arrott plot

(g)

FIGURE 3: SQUID measurement of $4 \% \mathrm{Mn}$-doped Ge grown on $\mathrm{Si}$, the left column is for the as-grown film and the right column is the film after $400^{\circ} \mathrm{C}$ annealing. (a) and (d) are the hysteresis loops at 10 and $298 \mathrm{~K}$; (b) and (e) are moment versus temperature curves; (c) and (f) are Arrott's plots. The red line in (f) shows the expected Arrott's plot at the Curie temperature. 


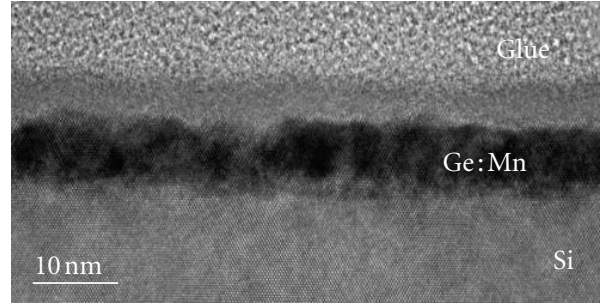

(a)

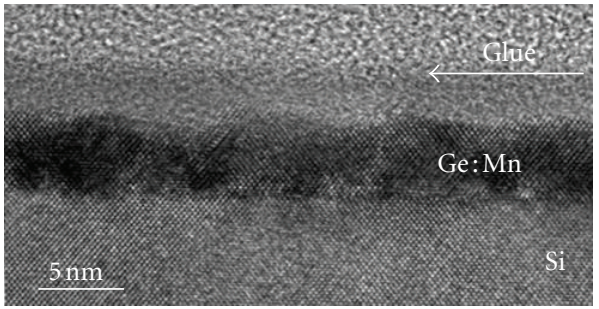

(b)

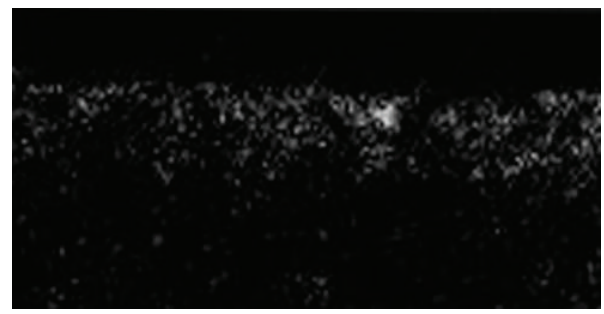

(c)

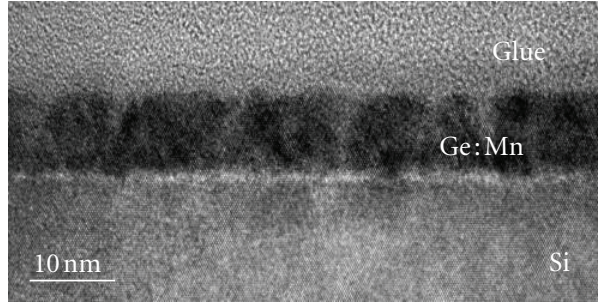

(d)

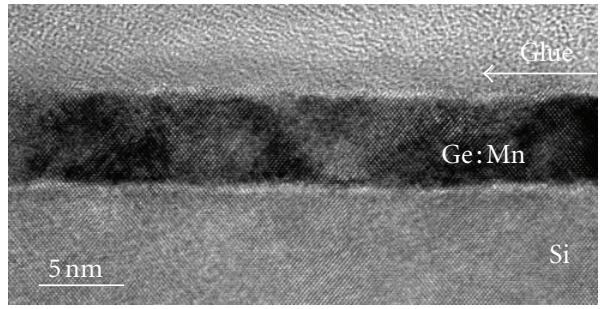

(e)

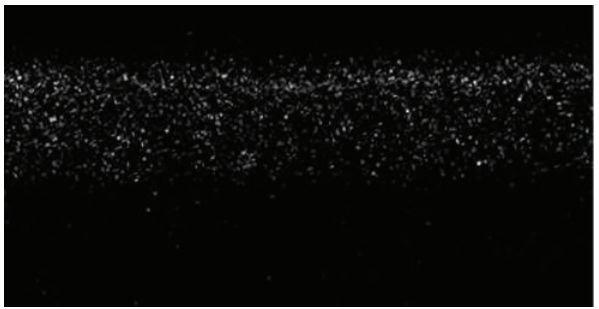

(f)

FIGURE 4: Typical TEM images of the as-grown (a) and the annealed (d) MnGe thin films with a thickness of $15 \mathrm{~nm}$; the left column is for the as-grown film and the right column is the film after $400^{\circ} \mathrm{C}$ annealing. (b) and (e) are the corresponding HRTEM images of (a) and (d), respectively; (c) and (f) EFTEM elemental maps of Mn correspond to the regions given by (a) and (d), respectively. The white arrows in (b) and (e) show the separation between the glue and the amorphous MnGe layer.

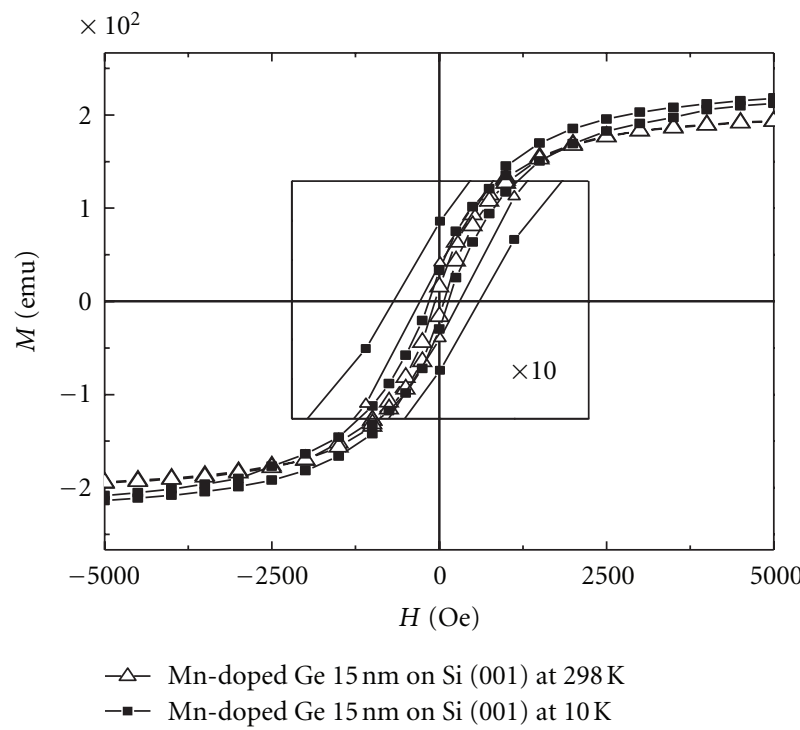

(a)

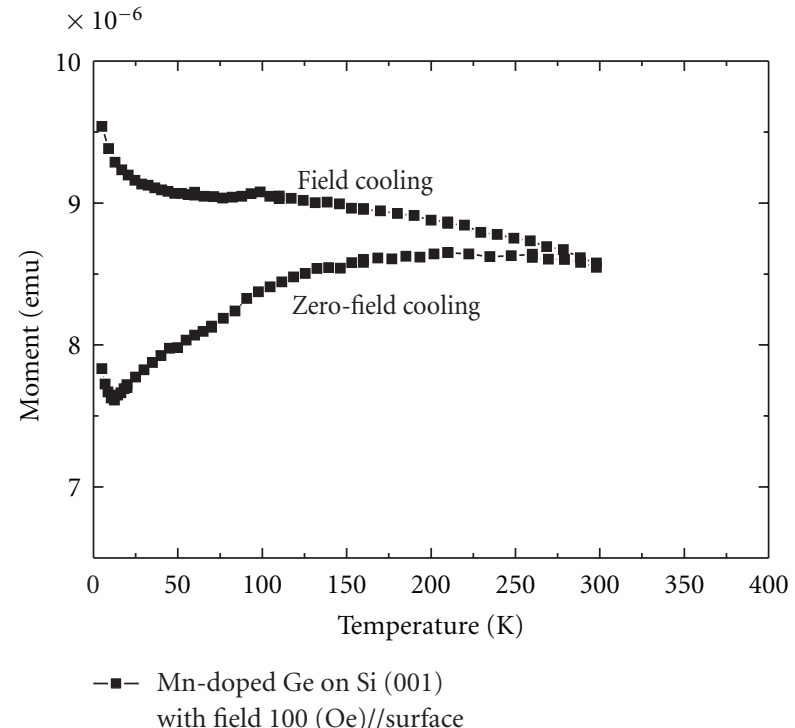

(b)

FIGURE 5: SQUID measurement of 4\% Mn-doped Ge thin film grown on Si with a thickness of $15 \mathrm{~nm}$. (a) Hysteresis loops measured at 10 and $298 \mathrm{~K}$; (b) magnetic moment versus temperature measured with a magnetic field of 100 Oe. 


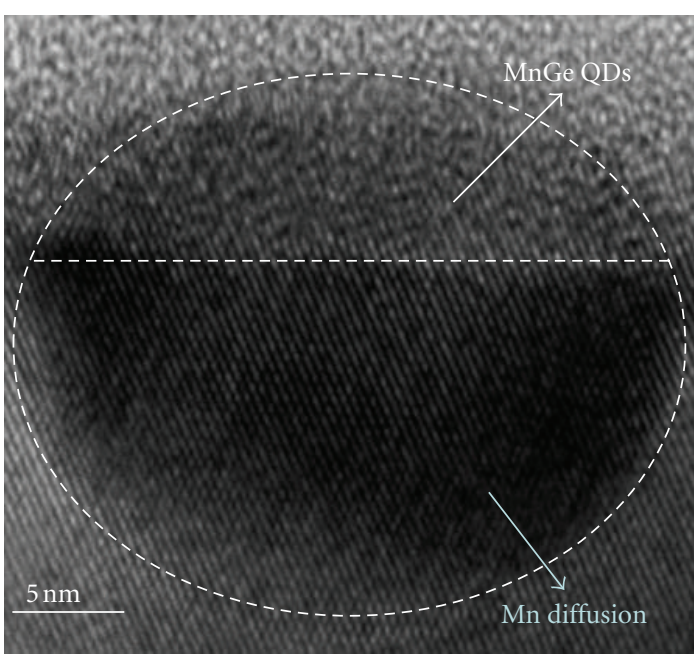

(a)

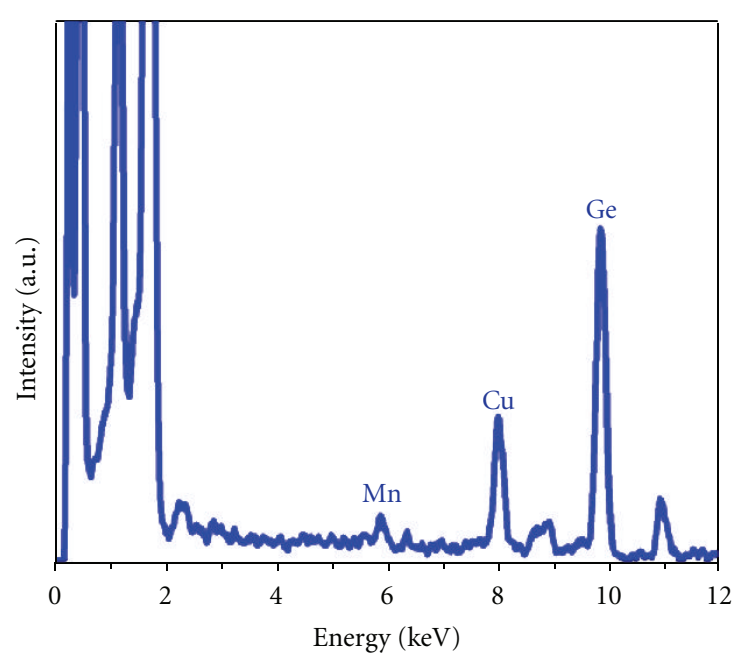

(b)

FIgURE 6: Structural properties of $\mathrm{Mn}_{0.05} \mathrm{Ge}_{0.95}$ quantum dots grown on a $p$-type Si substrate. (a) A high-resolution TEM cross-section image of typical $\mathrm{Mn}_{0.05} \mathrm{Ge}_{0.95}$ quantum dot showing the detailed lattice structure. (b) Typical EDS spectrum showing that both Mn and Ge are present in the $\mathrm{Mn}_{0.05} \mathrm{Ge}_{0.95}$ quantum dot. The average Mn concentration in the quantum dots was estimated to be $4.8 \pm 0.5 \%$.

moment as a function of temperature at a magnetic field of 100 Oe. The moment in the FC curve decreases very marginally as the temperature increases from 10 to $298 \mathrm{~K}$, showing that the $T_{c}$ is above the room temperature. The separation of the ZFC data from that of the FC shows a spin glass phase. Before and after annealing, there is no clear difference observed in magnetic properties. The $T_{c}$ can only be inferred to be above $400 \mathrm{~K}$ as shown in Figure $5(\mathrm{~b})$.

The difference of structural and magnetic properties between the $80 \mathrm{~nm}$ thick and the $15 \mathrm{~nm}$ thick $\mathrm{Mn}_{x} \mathrm{Ge}_{1-x}$ thin films suggest that when it comes to the nanostructures, the strain and the Mn distribution could be different from that of the bulk films. There might be a threshold thickness for a certain Mn concentration, beyond which the nucleation of metallic clusters (such as $\mathrm{Mn}_{5} \mathrm{Ge}_{3}$ and $\mathrm{Mn}_{11} \mathrm{Ge}_{8}$ ) takes place and accounts for the RT ferromagnetism $\left(T_{c}=296 \sim\right.$ $300 \mathrm{~K})[84,108]$. It is therefore of great interest to further investigate $\mathrm{Mn}_{x} \mathrm{Ge}_{1-x}$ nanostructures to explore their magnetic properties. $\mathrm{Mn}_{x} \mathrm{Ge}_{1-x}$ nanodots may be formed by ion implantation process of $\mathrm{Mn}$ into Ge and by a selfassembling process with MBE. Since the Mn ion implantation induces metallic clusters and defects [92], we will only focus on the $\mathrm{Mn}_{x} \mathrm{Ge}_{1-x}$ DMS QDs fabricated via MBE.

3.2.2. Self-Assembled DMS $M n_{x} G e_{1-x}$ QDs. $\mathrm{Mn}_{0.05} \mathrm{Ge}_{0.95}$ QDs were grown on $p$-type Si substrates. Cross-section TEM was carried out to determine the structural characteristics and the Mn composition. HR-TEM image reveals that a QD has a dome shape with a base diameter of about $30 \mathrm{~nm}$ and a height of about $8 \mathrm{~nm}$ (Figure 6(a)) The interface between the dot and the Si substrate has excellent lattice coherence. A careful inspection reveals that the dot is single crystalline without evidence of pronounced dislocations or stacking faults. However, because of the heavy Mn doping it is possible that some amount of point defects (such as Mn interstitials) may be present inside the dot, which is beyond the detect capability of conventional TEM.

Directly underneath the $\mathrm{Mn}_{0.05} \mathrm{Ge}_{0.95} \mathrm{QD}, \mathrm{Mn}$ diffuses into Si substrate and forms a strained $\mathrm{MnSi}$ area, which has the same diameter as the top $\mathrm{Mn}_{0.05} \mathrm{Ge}_{0.95} \mathrm{QD}$, but a height of about $16 \mathrm{~nm}$ (Figure 6(a)). This diffusion behavior is not unusual as it was also observed in (In, Mn)As and (In, Cr)As QDs systems [120, 121]. However, the migrations of $\mathrm{Mn}$ into the substrate make it difficult to accurately determine Mn concentration inside the dot. To address this challenge, we have performed energy dispersive X-ray spectroscopy (EDS) experiments (in a scanning TEM mode) to analyze the Mn composition at nanoscale (Figure 6(b)). When the EDS was performed, electron probes with several nanometers in diameter were illustrated on the $\mathrm{Mn}_{0.05} \mathrm{Ge}_{0.95}$ QDs and their underlying Si substrate. Consequently, Si peaks are constantly observed in the EDS spectra. To estimate the Mn concentrations, we performed a quantitative analysis of atomic percentages of $\mathrm{Mn}$ and $\mathrm{Ge}$ and artificially discounted the Si peak. The EDS analysis over many QDs reveals a $\mathrm{Mn} / \mathrm{Ge}$ atomic ratio of about $0.144: 1$. Since approximately $1 / 3$ volume fraction of $\mathrm{Mn}$ is distributed in the $\mathrm{Mn}_{x} \mathrm{Ge}_{1-x} \mathrm{QD}$ [40], the average $\mathrm{Mn}$ concentration can be estimated to be $4.8 \pm$ $0.5 \%$. Note that the deviation was determined by a thorough study of many $\mathrm{Mn}_{0.05} \mathrm{Ge}_{0.95}$ QDs. Both the HR-TEM investigations and the composition analysis suggest that each individual QD is a single crystalline DMS system.

Magnetic properties were studied using a superconducting quantum interference device (SQUID) magnetometer at various temperatures. Figure 7(a) and two corresponding insets show the temperature-dependent hysteresis loops when the external magnetic field is parallel to the sample surface (in-plane). The field-dependent magnetization indicates a strong ferromagnetism above $400 \mathrm{~K}$. The saturation magnetic moment per $\mathrm{Mn}$ atom is roughly estimated to be $1.8 \mu_{B}$ at $5 \mathrm{~K}$. A fraction of roughly $60 \%$ of $\mathrm{Mn}$ is estimated to be 


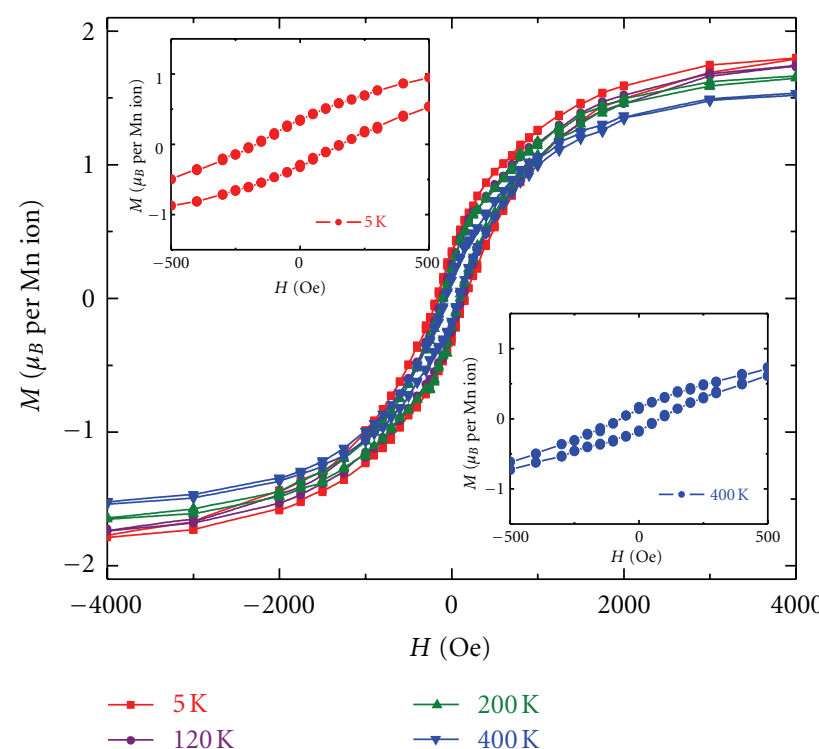

(a)

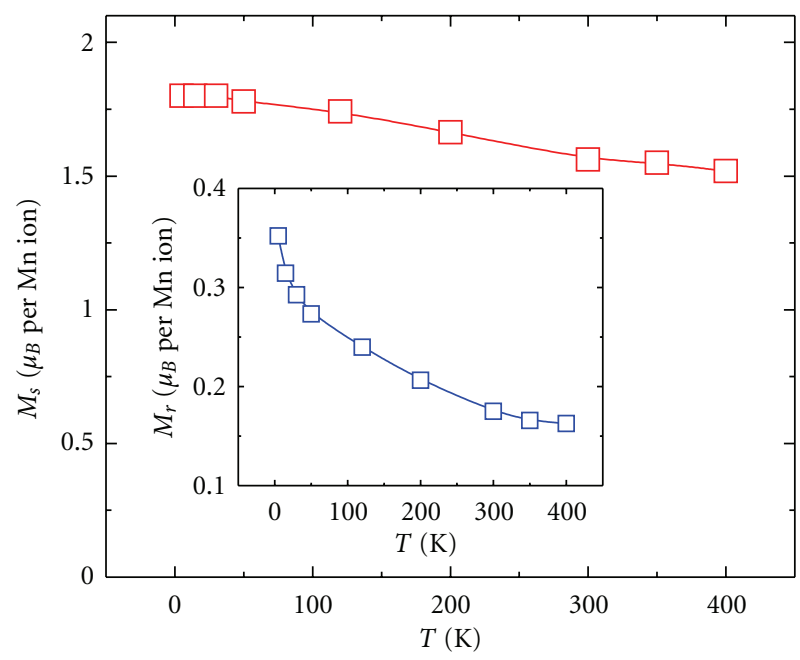

(c)

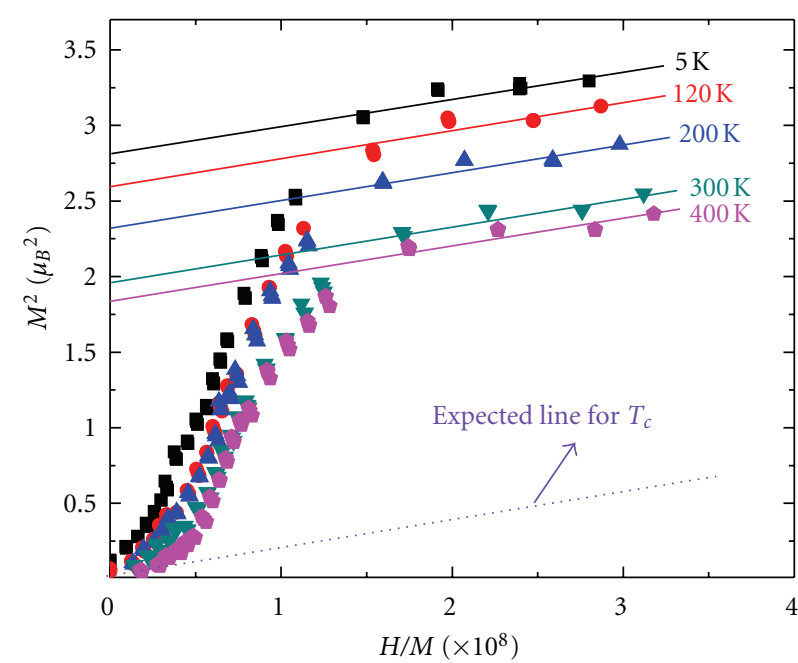

(b)

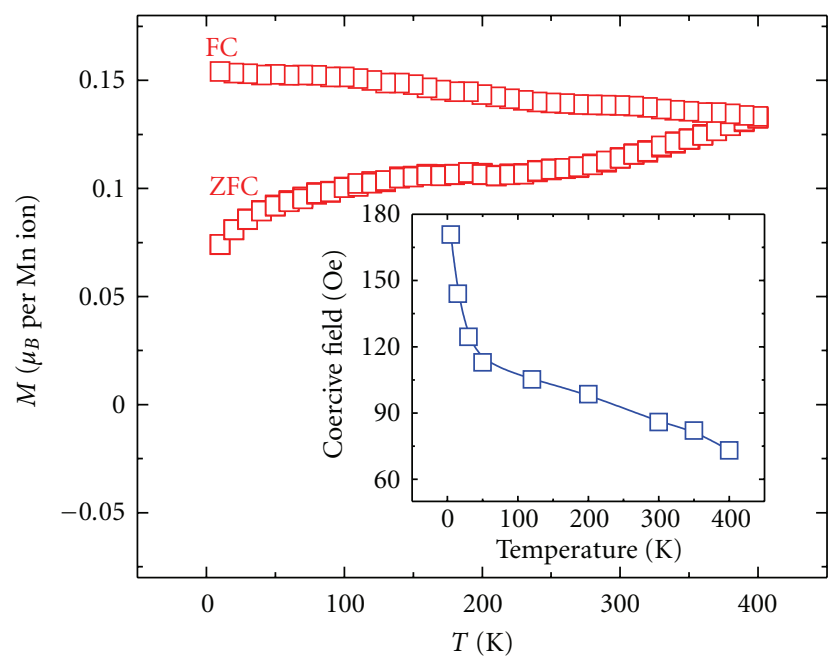

(d)

Figure 7: Magnetic properties of the $\mathrm{Mn}_{0.05} \mathrm{Ge}_{0.95}$ quantum dots grown on a $p$-type Si substrate. (a) Hysteresis loops measured at different temperatures from 5 to $400 \mathrm{~K}$. (b) The Arrott plots were made to obtain the Curie temperature. Consistent with (a), the Curie temperature is projected to be above $400 \mathrm{~K}$. (c) The temperature dependence of saturation moments. The inset gives the remnant moments with respect to temperature; (d) Zero-field cooled and field cooled magnetizations of quantum dots with a magnetic field of 100 Oe; the inset shows the coercivity values at different temperatures. The external magnetic field is in parallel with the sample surface.

activated assuming that each $\mathrm{Mn}$ has a moment of $3 \mu_{B}$ $[27,58]$. The Arrott plots were also made in order to evaluate the $T_{c}^{2}$ (Figure 7(b)). By neglecting the high-order terms, the magnetic field can be expressed in the following equation [122]:

$$
H=\frac{1}{\chi} M+\beta M^{3} \quad \text { or } \quad \frac{H}{M}=\frac{1}{\chi}+\beta M^{2},
$$

where $H$ is the external magnetic field, $M$ is the magnetic moment from the sample, $\chi$ is the susceptibility, and $\beta$ is a material dependent constant.

According to (1), $M^{2}$ can be plotted as a function of $H / M$. When $H / M$ is extrapolated to $M^{2}=0$, the intercept on the
$H / M$ axis gives $1 / \chi$. The $T_{c}$ for this material can be obtained when $1 / \chi$ vanishes. Figure $7(\mathrm{~b})$ shows the Arrott plots at several temperatures. It can be seen that even at $400 \mathrm{~K}$ the intercept $(1 / \chi)$ on the $H / M$ axis does not vanish, which means that the susceptibility still has a finite value and the $T_{c}$ has not been reached yet. By using the slope obtained at $400 \mathrm{~K}$, a dashed line can be drawn as shown in Figure 7(b), in which a $T_{c}$ is projected to be beyond $400 \mathrm{~K}$. This is in a good agreement with the data from the hysteresis loops showing the magnetic order above $400 \mathrm{~K}$. Figure $7(\mathrm{c})$ and corresponding inset show the temperature-dependent saturation and remnant moments per $\mathrm{Mn}$ ion, respectively. Both of them demonstrate weak temperature dependences and 
substantial amount of magnetization moments remain even at $400 \mathrm{~K}$.

ZFC and FC magnetizations were measured with a magnetic field of 100 Oe as shown in Figure 7(d). The magnetic moments do not drop to zero, suggesting a high $T_{c}$ beyond $400 \mathrm{~K}$, which is in a good agreement with the Arrott plots in Figure 7(b). From these two curves, one can also infer the formation of a single phase in this material system, that is, DMS QDs. The wide separation of the ZFC and FC curves in the temperature range of 5 to $400 \mathrm{~K}$ shows the irreversibility of susceptibilities, possibly arising from strain-induced anisotropy as a large lattice mismatch exists between $\mathrm{Si}$ and Ge [61]. The temperature-dependent coercivity is shown in Figure 7 (d) inset. The coercivity decreases from 170 Oe (at $5 \mathrm{~K}$ ) to $73 \mathrm{Oe}$ (at $400 \mathrm{~K}$ ). The small coercivity in the entire temperature range measured features a soft ferromagnetism which originates from $\mathrm{Mn}$ ions diluted in the Ge matrix [30]. The above magnetic properties support the fact that the $\mathrm{Mn}_{0.05} \mathrm{Ge}_{0.95}$ QDs exhibit a DMS-type ferromagnetic order.

It is well known that the direct interaction (superexchange) between $d$-shells of the adjacent Mn atoms leads to an antiferromagnetic configuration of the $d$-shell spins [8]. Therefore, the presence of $\mathrm{Mn}-\mathrm{Mn}$ clusters would lead to the antiferromagnetism; but when this pair is surrounded by other Mn atoms, the ferri- or ferromagnetic coupling may appear. However, the extensive high-resolution TEM did not reveal observable Mn clusters although such a possibility cannot be completely excluded (Figure 6). Thus, the origin of strong ferromagnetism possibly comes from the $\mathrm{Mn}_{0.05} \mathrm{Ge}_{0.95}$ QDs via the hole mediation process. In general, when Mn dopants are introduced into Ge, they produce two acceptor levels, that is, $0.16 \mathrm{eV}$ and $0.37 \mathrm{eV}$ above the valence band [123]. When the doping concentration becomes high, the impurity bands could be developed (instead of being separate acceptor levels). However, theoretically the formation of such impurity bands may not affect the physics of ferromagnetism [124]. In the following, we will analyze the Mn-doping behavior by considering a single acceptor level of $0.16 \mathrm{eV}$ in an approximate form. At a sufficiently high temperature, $\mathrm{Mn}$ acceptors can be activated to generate holes. These holes are itinerant among a number of $\mathrm{Mn}$ ions and align $\mathrm{Mn}$ local spins along one direction via the $p-d$ exchange coupling to reduce the total energy of the system, resulting in the hole-mediated ferromagnetism [125]. Experimentally this phenomenon has been widely observed in several material systems, such as group III-V (GaAs and InAs) [2, 126], group II-VI ( $\mathrm{ZnO})$ [127], and group IV (Ge) [27, 39]. While most of research focuses on bulk DMS, of particular interest is to study the ferromagnetism origin in quantum structures such as (In, Mn)As and $\mathrm{Mn}_{x} \mathrm{Ge}_{1-x}$ QDs. Previously, Chen et al. [39] and others [120] have suggested that the carrier confinement in a DMS QD can strengthen hole localization and subsequently enhance the thermal stability of magnetic polarons giving rise to a higher $T_{c}$ than bulk films.

Atomic force microscopy (AFM) and magnetic force microscopy (MFM) measurements were carried out to investigate the morphology and ferromagnetism of $\mathrm{Mn}_{0.05} \mathrm{Ge}_{0.95}$ QDs at $320 \mathrm{~K}$, respectively. The average dot size is $50 \mathrm{~nm}$ in base diameter and $6 \mathrm{~nm}$ in height. The dot density is about
$6 \times 10^{9} \mathrm{~cm}^{-2}$ (Figure $8(\mathrm{a})$ ). The corresponding MFM image was taken by lifting up the MFM probe $25 \mathrm{~nm}$ above the topographic height of the sample in a phase detection mode (Figure $8(\mathrm{~b})$ ). The appearance of bright-and-dark areas in the MFM image clearly shows the formation of magnetic domains in the $\mathrm{Mn}_{0.05} \mathrm{Ge}_{0.95}$ QDs, which is similar to (In, Mn)As DMS QDs [128]. Figures 8(c)-8(f) show enlarged MFM images of several individual $\mathrm{Mn}_{0.05} \mathrm{Ge}_{0.95}$ QDs. By reversing the tip magnetization, opposite contrast was observed for each dot, indicating that the magnetic signals originated from the top $\mathrm{Mn}_{0.05} \mathrm{Ge}_{0.95}$ QDs. Note that each QD is a single domain "particle." During the magnetization process, the domain would rotate preferentially along the magnetic field to produce net magnetization moments. Since the experiments were performed at $320 \mathrm{~K}$, the formation of metallic phases such as $\mathrm{Mn}_{5} \mathrm{Ge}_{3}$ and $\mathrm{Mn}_{11} \mathrm{Ge}_{8}$ can be easily ruled out because they have low Curie temperatures of 296 300 K. Overall, the above MFM results agree well with the TEM observations and the ferromagnetic order at high temperature obtained in the SQUID measurements.

3.3. Discussions. We have reviewed the growth of $\mathrm{Mn}_{0.05} \mathrm{Ge}_{0.95}$ by MBE, ion implantation, and bulk sintering, emphasizing the growth temperature effect on the phase formation and magnetic properties. While the current motivation is driven by the prospect of enhancing Curie temperatures, the experiments show a strong tendency of metallic precipitates developed in bulk and relatively thick $\mathrm{Mn}_{x} \mathrm{Ge}_{1-x}$ crystals. The fundamental understanding here points to a spin-glasslike feature in $\mathrm{Mn}_{x} \mathrm{Ge}_{1-x}$ due to the formation of various lattice coherent nanostructures and metallic precipitates, which exhibit magnetic blocking behaviors under different temperature regimes. The challenge to eliminate these clusters seems to be overwhelming and nearly impossible. However, when the material dimension decreases, it evidences a change of material structures being free of precipitates and a significant increase of Curie temperatures over $400 \mathrm{~K}$. The preliminary explanation on these behaviors can be attributed to the carrier confinement in a DMS QD which strengthens hole localization and subsequently enhances the thermal stability of magnetic polarons giving rise to a higher $T_{c}$ than those of bulk films. Nevertheless, the experimental data suggest that with nanoscale structures, the quantum confinement effect comes into being which significantly influences the exchange coupling between the confined holes and the localized $\mathrm{Mn}^{2+}$. Future progress in this direction will involve a detailed theoretical treatise to understand the quantum confinement effect on magnetic properties in a quantitative picture.

\section{Electric-Field-Controlled Ferromagnetism}

4.1. Introduction. Electric field control of ferromagnetism has a potential to realize spin field-effect transistors (spin FETs) and nonvolatile spin logic devices via carrier-mediated effect $[129,130]$. With the manipulation of carrier spins, a new generation of nonvolatile (green) computing systems could be eventually developed for many low-power-dissipation applications in all fields, including sensor network, 


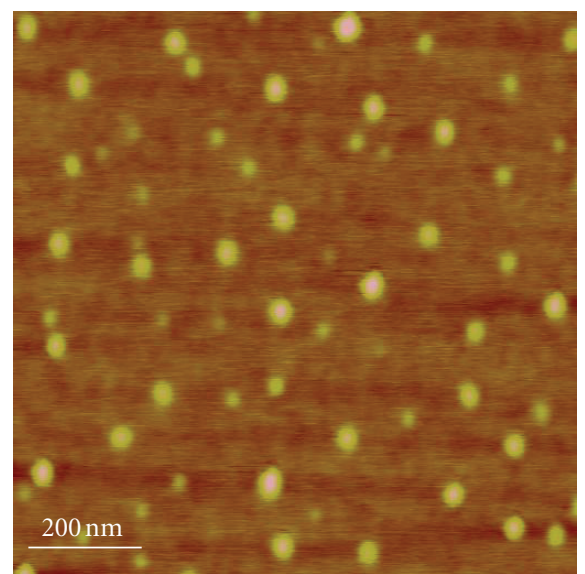

(a)
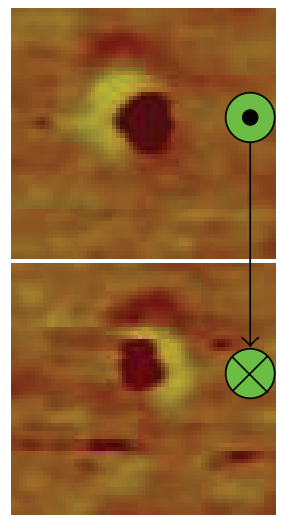

(c)

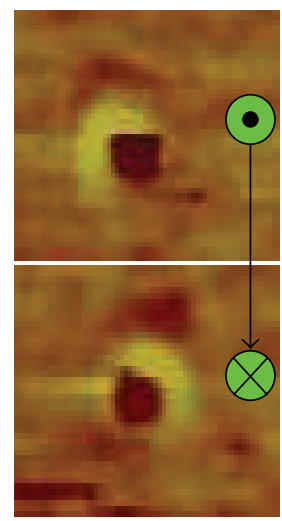

(d)

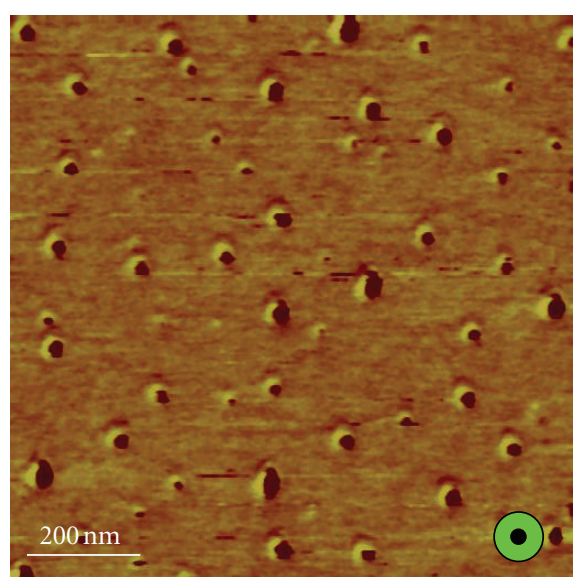

(b)

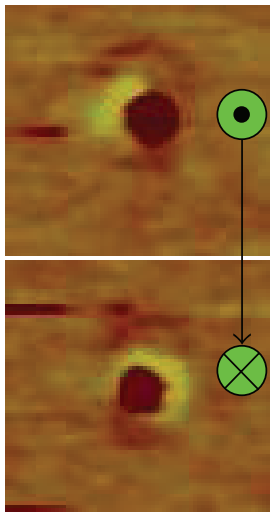

(e)

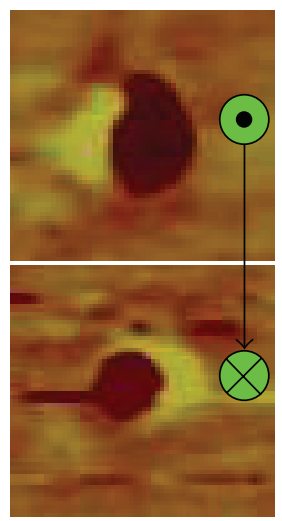

(f)

Figure 8: AFM and MFM images of the $\mathrm{Mn}_{0.05} \mathrm{Ge}_{0.95}$ quantum dots measured at $320 \mathrm{~K}$. (a) Typical AFM image of $\mathrm{Mn}_{0.05} \mathrm{Ge}_{0.95}$ quantum dots; (b) corresponding MFM image with the tip magnetization pointing toward the sample; (c) (f) enlarged MFM images of individual quantum dots taken from (b). From these MFM measurements, opposite contrasts were observed when applying an opposite magnetization to the tip. The results suggest that the magnetic signals come from the $\mathrm{Mn}_{0.05} \mathrm{Ge}_{0.95}$ quantum dots. The formation of $\mathrm{Mn}_{5} \mathrm{Ge}_{3}$ and $\mathrm{Mn}_{11} \mathrm{Ge}_{8}$ can be excluded because of their low Curie temperatures of $296 \sim 300 \mathrm{~K}$.

health monitoring, information, and sustainable wireless system. Since Datta and Das [131] first introduced the concept of spin FETs in 1990, enormous efforts were dedicated to creating a device wherein the carrier transport is modulated by electrostatic control of carrier spins [2, 20, 22, 23, 132136]. To understand and exploit this controllability, several theoretical models were proposed to explain the ferromagnetic coupling in DMS on a microscopic scale (also elaborated in the theoretical section): the Zener Kinetic-exchange [10], double-exchange [137], and the RKKY interaction $[138,139]$. These models share a common feature that a spontaneous ferromagnetic order is carrier-mediated through the increase of carrier concentrations. One of major challenges, however, is to search an ideal material with room-temperature controllable spin states $[21,22,140]$. In recent years, emerged DMSs became one of the promising candidates since they could possibly offer high $T_{c}$ in excess of $300 \mathrm{~K}$ [137]. The demonstration of the carrier-medicated ferromagnetism involving correlated electron/hole systems leads to a para- to ferromagnetism phase transition $[27,39$, $130,137]$. In principle, the collective alignment of spin states in these DMSs can be manipulated by the modulation of carrier concentrations through gate biasing in a FET structure $[2,140]$. For this kind of spin FETs, the "source" and "drain" may be completed through "nanomagnet," which are in turn controlled by the gate; and no carrier transport is needed. Clearly, one may also involve the control of source-drain conductance by gate-voltage-induced precession of injected spins (from the source). Since early 2000s, a significant progress was achieved $[2,23,130,135]$, in which the ferromagnetism of a (In, Mn)As channel layer could be effectively turned on and off via electric fields in a gated FET. Such extraordinary field-modulated ferromagnetism immediately rendered the development of future spintronic devices. However, the manipulation of ferromagnetism was limited because of low $T_{c}$ of the Mn-doped III-V materials [141]. Therefore, a search for new DMS materials with $T_{c}>300 \mathrm{~K}$ and carrier-mediated ferromagnetism becomes a current global challenge $[10,140]$.

4.2. Electric-Field-Controlled Ferromagnetism in Mn-Implanted Ge Dots. In parallel with the experimental efforts on field 

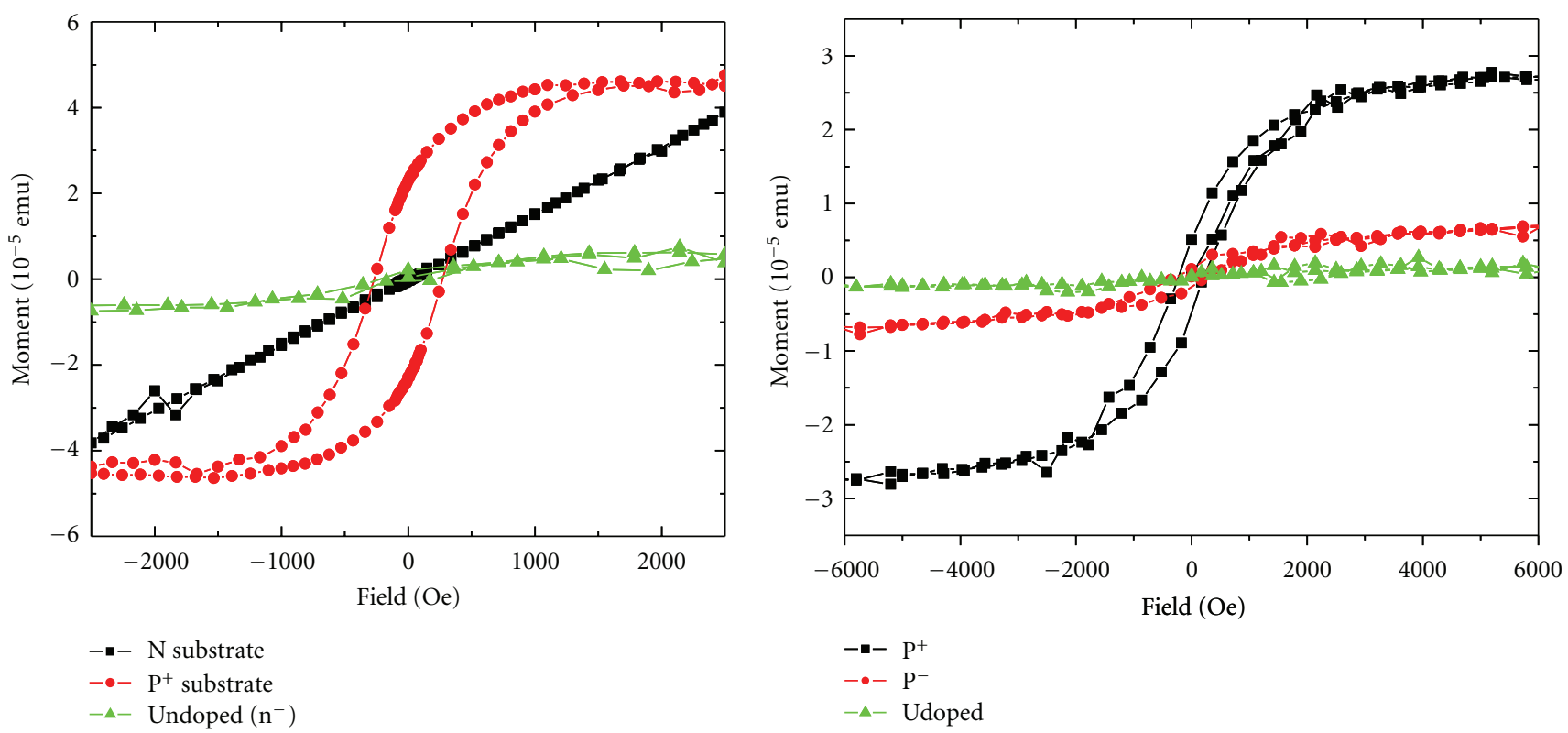

Figure 9: The magnetic properties of Mn-implanted Ge via a nanopatterned $\mathrm{SiO}_{2}$ mask. The hole-mediated effect can be clearly observed in the Mn-implanted p-type Ge sample.

controlled ferromagnetism in III-V material system, we have previously investigated the magnetic properties of $\mathrm{Mn}_{x} \mathrm{Ge}_{1-x}$ nanostructures by ion implantation of $\mathrm{Mn}$ [39]. We demonstrated the hole-mediated effect in Mn-implanted nanodot structures and the modulation of their ferromagnetism by applying gate biases in MOS capacitors at a low temperature of $10 \mathrm{~K}$. Figure 9 shows the magnetic properties of samples with Mn implantation into Ge via the nanopatterned $\mathrm{SiO}_{2}$ mask on different doped substrates. It is observed that the Mn-implanted $p^{+} \mathrm{Ge}\left(10^{19} \mathrm{~cm}^{-3}\right)$ has stronger ferromagnetic signals than that $n$-type $\mathrm{Ge}$ $\left(10^{17} \mathrm{~cm}^{-3}\right)$, which may indicate a hole-mediated effect. Note that we cannot completely exclude the possibility of the formation of dopant complexes, such as B-Mn and Sb-Mn in the $p^{+}$and $n$-type Ge, respectively. Taking advantage of this effect, we further fabricated a MOS capacitor device with $\mathrm{Mn}$-implanted Ge as the channel layer. Figure 10 shows the magnetic moment versus applied field for the MOS structure on the $n$-type substrate under various gate biases at $10 \mathrm{~K}$. When the voltage is applied from -6 to $-30 \mathrm{~V}$, the hole concentration in the $\mathrm{Mn}_{x} \mathrm{Ge}_{1-x}$ channel increases and the magnetic hysteresis becomes larger. At $0 \mathrm{~V}$ bias, only a line with a small slope and a small negligible loop are seen; this is because a low density of excess holes is present. When a positive voltage is applied to the gate, holes are partially depleted, resulting in a decrease of the channel magnetization and in the disappearance of magnetic hysteresis. These experimental results demonstrated the hole-mediated effect in $\mathrm{Mn}_{x} \mathrm{Ge}_{1-x}$, consistent with the finding by Park et al. [27]. However, the metallic precipitates such as $\mathrm{Mn}_{5} \mathrm{Ge}_{3}$ and $\mathrm{Mn}_{11} \mathrm{Ge}_{8}$ and implantation damages were found in these nanostructures, which made the system rather complex and might have partly jeopardized the hole-mediated effect.

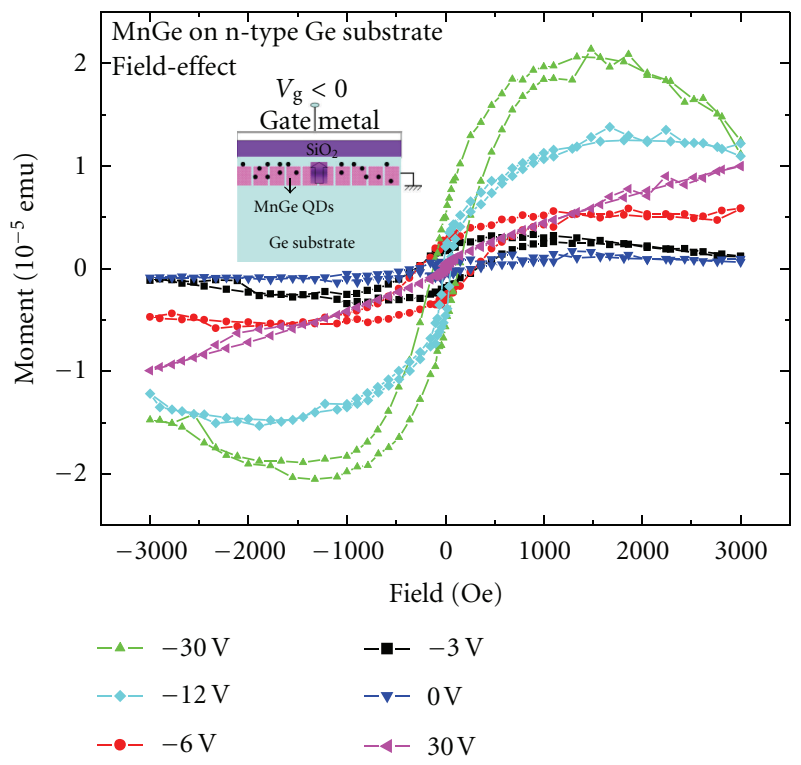

FIGURE 10: Magnetic moment versus applied field under various biases from -30 to $30 \mathrm{~V}$ at $10 \mathrm{~K}$. The obtained hysteresis is the largest under $-30 \mathrm{~V}$ gate voltage, and the ferromagnetic phase is turned off under $+30 \mathrm{~V}$ gate bias. The inset shows the spin-gated structure used to investigate $C$ - $V$ and gate-bias-dependent magnetic properties.

4.3. Electric-Field-Controlled Ferromagnetism in Self-Assembled DMS $M n_{0.05} \mathrm{Ge}_{0.95}$ QDs. Since the self-assembled $\mathrm{Mn}_{0.05} \mathrm{Ge}_{0.95}$ QDs have a high $T_{c}$ above $400 \mathrm{~K}$ and are free of metallic phases $\left(\mathrm{Mn}_{5} \mathrm{Ge}_{3}\right.$ and $\left.\mathrm{Mn}_{11} \mathrm{Ge}_{8}\right)$, it can be potentially 


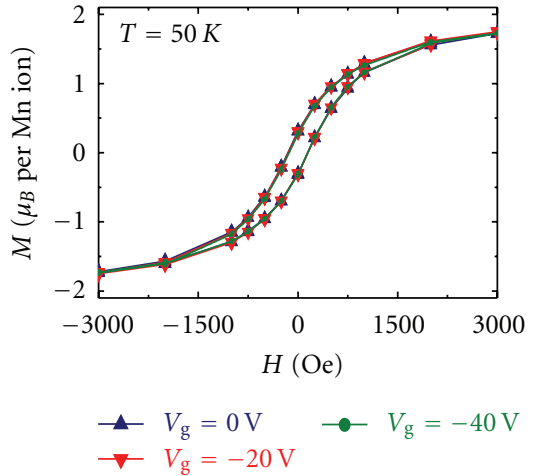

(a)
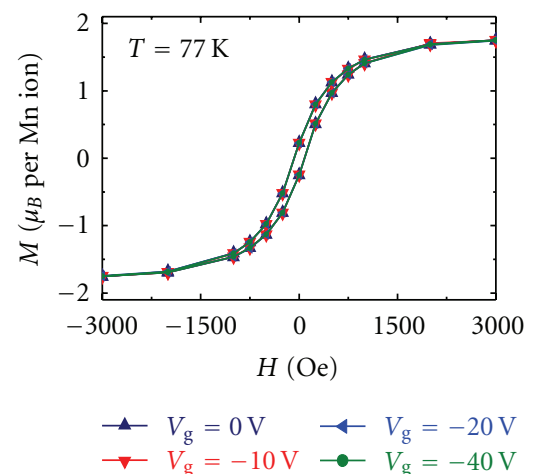

(d)

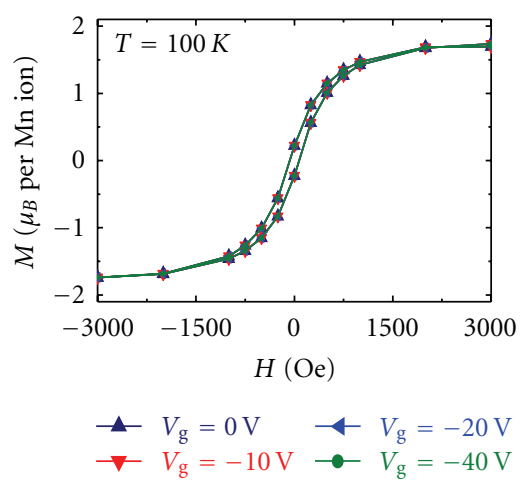

(g)

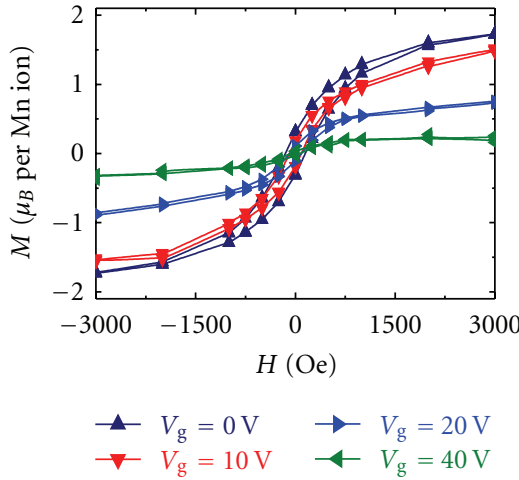

(b)
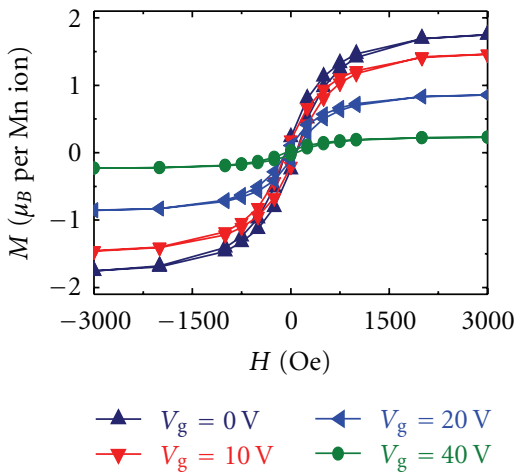

(e)

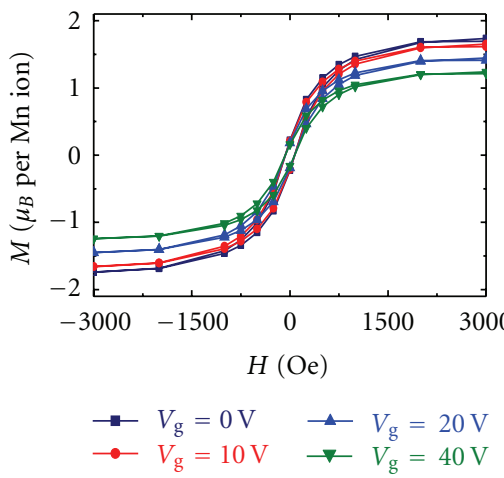

(h)

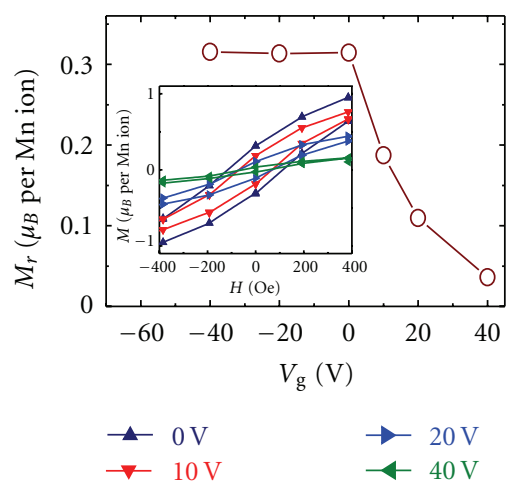

(c)

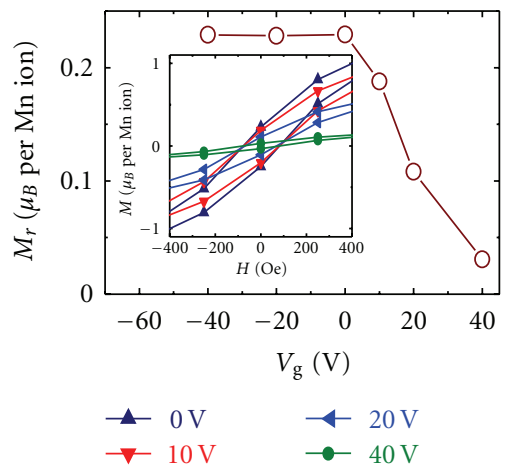

(f)

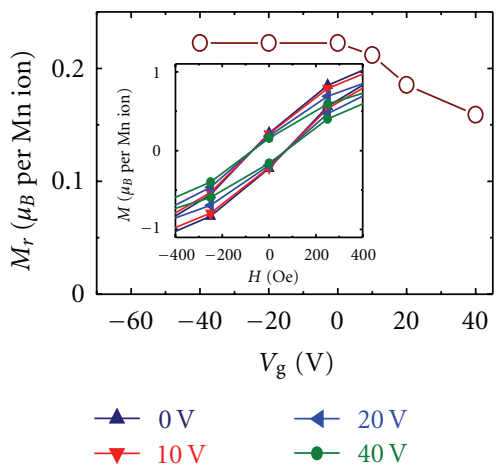

(i)

Figure 11: Control of ferromagnetism of $\mathrm{Mn}_{0.05} \mathrm{Ge}_{0.95}$ quantum dots by applying electric field at $50 \mathrm{~K}((\mathrm{a}) \sim(\mathrm{c}))$, $77 \mathrm{~K}((\mathrm{~d}) \sim(\mathrm{f}))$, and $100 \mathrm{~K}$ $((\mathrm{g}) \sim(\mathrm{i}))$. (a), (d), and (g) Hysteresis loops with zero and negative bias of $-10,-20$ and $-40 \mathrm{~V}$ on the gate; (b), (e), and (h) the hysteresis loops with zero and positive bias of $+10,+20$, and $+40 \mathrm{~V}$; (c), (f), and (i) remnant moments with respect to the gate bias. Insets of (c), (f) and (i) are enlarged figures from the central part of (b), (e), and (h) to clearly show the change of remnant moments, respectively. It is found that both the saturation and remnant moments can be manipulated by applying biases on the MOS gate at 50, 77, and $100 \mathrm{~K}$.

used as a channel layer in a MOS device to study the modulation of ferromagnetism by electric field. The device structure consists of a metal gate $(\mathrm{Au}, 200 \mathrm{~nm}), \mathrm{Al}_{2} \mathrm{O}_{3}$ $(40 \mathrm{~nm}), \quad \mathrm{Mn}_{0.05} \mathrm{Ge}_{0.95}$ QDs $(6 \mathrm{~nm})$, a wetting layer $(<0.6 \mathrm{~nm})$, a $p$-type $\mathrm{Si}$ substrate $\left(1 \times 10^{18} \mathrm{~cm}^{-3}\right)$, and a back metal contact (Au, $200 \mathrm{~nm})$. A relative thick dielectric of $\mathrm{Al}_{2} \mathrm{O}_{3}(40 \mathrm{~nm})$ is employed to ensure a small leakage current to a level below $10^{-6} \mathrm{~A} / \mathrm{cm}^{2}$. Figure 11 shows the electric field controlled ferromagnetism performed at 50,77 , and $100 \mathrm{~K}$, corresponding to (a)-(c), (d)-(f), and (g)-(i), respectively. Due to the similarity of the data, we take $77 \mathrm{~K}$ as an example to describe the device operation in Figures 11(d)-11(f). Figures 11(d) and 11(e) show the hysteresis loops by SQUID with negative and positive biases on the MOS gate at $77 \mathrm{~K}$, respectively. Under a negative bias, the holes are attracted into the channel of the device (accumulation). In this circumstance, however, the hysteresis loop does not show a remarkable change (Figure 11(d)). This could be explained 
by the fact that even at $0 \mathrm{~V}$, the QDs device is already accumulated with enough holes to induce ferromagnetism. In other words, the hole-mediated effect is sufficient to align a majority of the activated $\mathrm{Mn}$ ions along one direction in each individual QD. Further increasing negative bias does not change much on the hole concentrations. On the contrary, with the positive bias, a large amount of holes are depleted into the $p$-type Si so that hole-mediated effect is notably reduced. In fact, the surface of the device can have a high concentration of electrons if the leakage is limited (inversion). As a result, the $\mathrm{Mn}$ ions start to misalign because of the lack of holes. The saturation moment per $\mathrm{Mn}$ ion decreases more than 10 times as the gate bias increases from 0 to $+40 \mathrm{~V}$ (Figure 11(e)). It should be noted that, at $+40 \mathrm{~V}$, the saturation and remnant moments of the $\mathrm{Mn}_{0.05} \mathrm{Ge}_{0.95}$ QDs become fairly weak, resembling a "paramagnetic-like" state. Figure 11(f) summarizes the change of remnant moments as a function of gate voltage. A similar trend in the carrier density as a function of the gate bias was observed from CV curves (not shown here), suggesting a strong correlation between hole concentrations and the ferromagnetism, that is, the hole-mediated effect. The inset in Figure 11(f) displays an enlarged picture to clearly show the change of remnant moments with respect to the gate bias. By increasing the measurement temperature to $100 \mathrm{~K}$ (Figures 11(g)-11(i)), the modulation of the ferromagnetism becomes less pronounced compared to those at 50 and $77 \mathrm{~K}$ due to the increased leakage current in our MOS devices. The above results evidently demonstrate that the hole-mediated effect does exist in this material system.

4.4. Discussions. Electrical-field-controlled ferromagnetism has been successfully demonstrated in Mn-doped III-V DMSs $[2,23,130,135,140]$. It is well established that the variation of hole concentrations renders such a controllability [2]. The low $T_{c}$ of III-V DMS, however, presents a challenge to further realize room-temperature-controlled ferromagnetism [141]. Alternatively, the recent experiments on $\mathrm{Mn}_{0.05} \mathrm{Ge}_{0.95}$ QDs show a high $T_{c}$ above room temperature and gate-modulated ferromagnetism over $100 \mathrm{~K}$ [40]. While the leakage current suppressed the gate modulation, room-temperature-controlled ferromagnetism would not be impossible because of the high $T_{c}$ of this system. Research in this direction may open up a pathway for achieving roomtemperature Ge-based (and other) spin-FETs and spin logic devices. These spintronic devices could potentially replace the conventional FETs with lower power consumptions and provide additional new device functionalities, which is beyond today's mainstream CMOS technology of microelectronics.

\section{Metallic $\mathbf{M n}_{x} \mathbf{G e}_{1-x}(x \sim 20 \%)$ Nanodot Arrays}

5.1. Introduction. There is also a need to develop ferromagnet/semiconductor hybrid structures for semiconductor spintronics since they have magnetic and spin-related functions and excellent compatibility with semiconductor device structures [142, 143]. By embedding magnetic nanocrystals into conventional semiconductors, a unique hybrid system can be developed, allowing not only utilizing the charge properties but also the spin of carriers, which immediately promises next-generation nonvolatile magnetic memories and sensors $[144,145]$. On the other hand, spin injections into the semiconductor can be dramatically enhanced via coherent nanostructures, which considerably reduce undesired spin scatterings [146]. Although magnetic hybrid systems, such as MnAs/GaAs, have been extensively studied over several decades, the control (over the spatial location, shape and geometrical configuration) of the magnetic nanostructures (for instance MnAs) still remains a major challenge to further improve the performance of the related magnetic tunnel junctions (MTJs) and spin valves [147].

5.2. Growth Methods. We employed a concept of stacked $\mathrm{Mn}_{x} \mathrm{Ge}_{1-x}(x \sim 20 \%)$ (hereafter, we denote it as MnGe) nanodots by alternatively growing $\mathrm{MnGe}$ and Ge layers with designated thicknesses (nominal $3 \mathrm{~nm}$ thick MnGe and $11 \mathrm{~nm}$ thick Ge). It is well known that $\mathrm{Mn}$ doping in $\mathrm{Ge}$ induces compressive strain because of its larger atomic size [148], assuming that no lattice defects are generated during the doping process, that is, lattice coherence. Mn-rich MnGe nanodots induced by the spinodal decomposition should be strained if the lattice coherence between the nanodots and the matrix remains. Once the strained nanodots are developed, a thin Ge spacer layer, subsequently deposited with an optimized thickness, will retain the perfect lattice coherence with the underneath nanodots. This enables the existing nanodots to exert strain on the Ge spacer layer and produce "strained spots," which, in turn, become preferred nucleation sites for successive nanodots. Eventually, multilayered and vertically aligned MnGe nanodot arrays can be produced, similar to the scenarios of stacked InAs/GaAs $[149,150]$ and $\mathrm{Ge} / \mathrm{Si}[151]$ quantum dots. Indeed, by employing this innovative approach, we achieved the growth of coherent selfassembled MnGe nanodot arrays with an estimated density of $10^{11} \sim 10^{12} \mathrm{~cm}^{-2}$ within each MnGe layer.

Ten periods of MnGe nanodots were epitaxially grown on $\mathrm{Ge}(100)$ and GaAs (100) substrates by the MBE system. TEM and EDS in the scanning TEM (STEM) mode were performed to understand the nanostructures and compositional variations of the resulting thin films. Figures 12(a) and 12(c) are typical plane-view and cross-sectional TEM images and show the general morphology of the MnGe nanostructures, viewed along the $\langle 100\rangle$ and $\langle 011\rangle$ directions, respectively. A high density of dark nanodots can be clearly seen in both cases. Based on the magnified cross-sectional image shown in Figure 12(d), the nanodot arrays are clearly observed with 10 stacks along the growth direction although not perfectly vertical. In order to determine the composition of the dark dots, EDS analyses in the STEM mode were carried out and typical plane-view and cross-sectional STEM images are shown in Figures 12(b) and 12(e), respectively. Figure 12(f) is the EDS result taken from a typical dot and shows clearly the $\mathrm{Mn}$ and Ge peaks. Figures 12(g) and 12(h) present EDS line scans using the Mn $K$ peak for the dots marked by $\mathrm{G}$ and $\mathrm{H}$ in Figures 12(b) and 12(e), respectively, indicating high concentrations of $\mathrm{Mn}$ inside the dots. Taking all these comprehensive TEM results into account, it is concluded that 


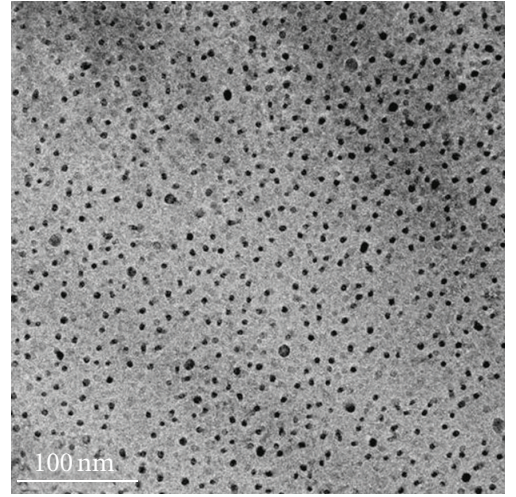

(a)

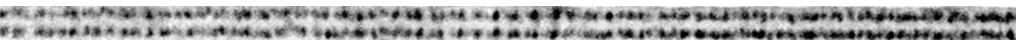

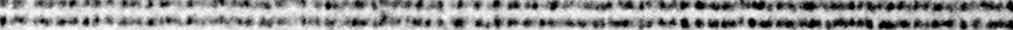
wa kes.

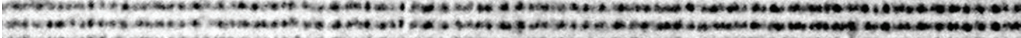

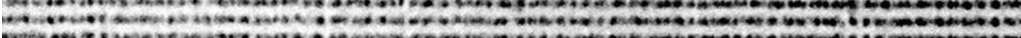

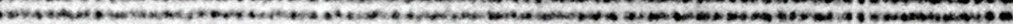

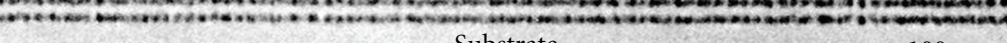
Substrate

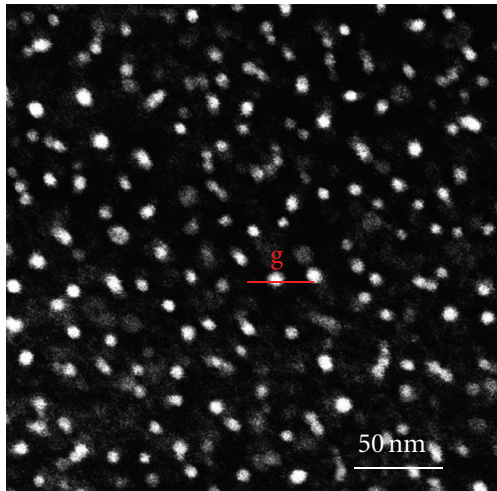

$100 \mathrm{~nm}$

(c)

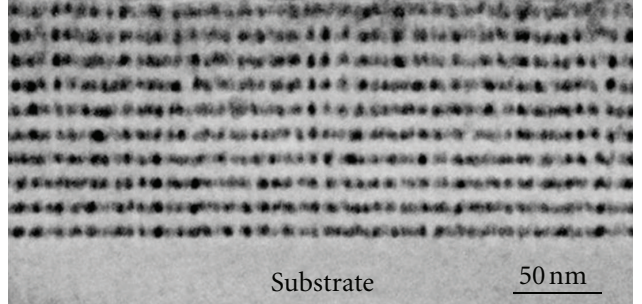

(d)

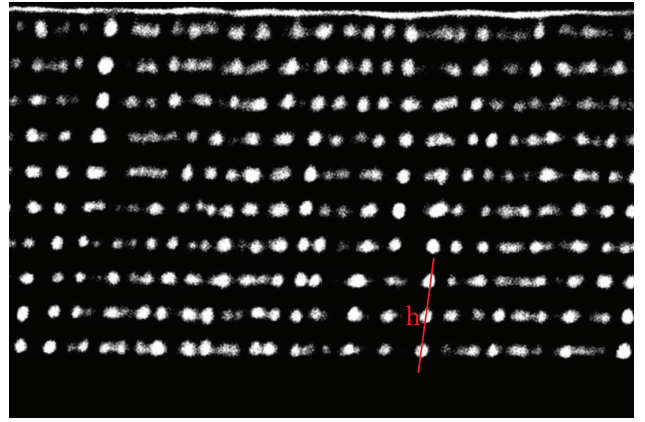

(e)

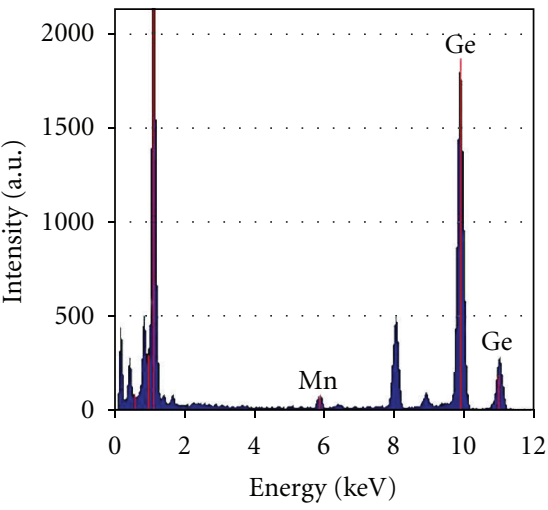

(f)

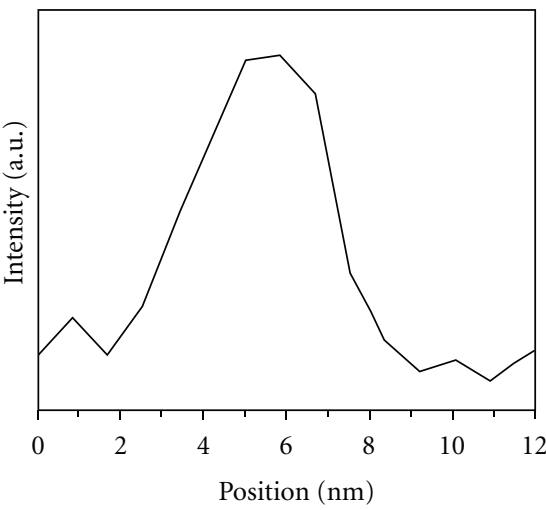

(g)

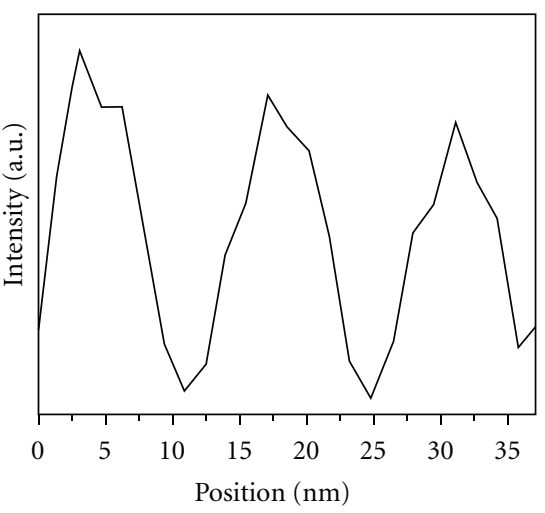

(h)

FIgURE 12: Transmission electron microscopy (TEM), scanning TEM, and energy dispersive X-ray spectroscopy (EDS) results of the multilayer MnGe nanodots. (a) A typical low-magnification plane-view bright-field TEM image showing MnGe nanodots (dark spots). (b) A plane-view low-angle dark-field STEM image showing the MnGe nanodots (white spots). (c) A low-magnification cross-sectional bright-field TEM image showing the obtained MnGe nanodot array in a large area. (d) A higher-magnification cross-sectional TEM image and (e) a cross-section STEM image, both showing the MnGe nanodot arrays. (f) An EDS profile showing the Mn and Ge peaks. (g, h) EDS line-scan profiles of the marked line in (b) and (e) using Mn $K$ peak, respectively, confirming nanodots being Mn rich. All TEM images are taken from the same sample.

the nanodots are Mn rich when compared with the surrounding matrix.

Figure 13(a) shows a high-magnification TEM image taken from a thin area, where several aligned MnGe nanodots can be evidently observed. The distance between two vertically adjacent nanodots (along the growth direction) is measured to be $14 \pm 1 \mathrm{~nm}$, well matched with the designed period of a $11 \mathrm{~nm}$ thick Ge spacer layer and a $3 \mathrm{~nm}$ thick MnGe layer. It should be noted that these nanodots are uniform in size with an elliptical shape (a dimension of $5.5 \pm 0.5 \mathrm{~nm}$ and $8 \pm 0.3 \mathrm{~nm}$ in the horizontal and vertical directions, resp.), as demonstrated in Figure 13(a). Since the nominal thickness of the MnGe layer $(3 \mathrm{~nm})$ is far less than the dot vertical dimension $(8 \mathrm{~nm})$, it suggests that, during the growth of the MnGe thin film, Mn not only diffuses laterally (to form dots), but also migrates vertically into the adjacent Ge spacer layers, primarily in the proximity of the dot regions, resulting in ellipse-shaped nanodots.

5.3. Magnetotransport Properties of Nanodot Arrays. The resistivity measurements were carried out to probe the carrier transport under different temperatures. It was found that the temperature-dependent resistivities rapidly increase with decreasing temperature due to the carrier freeze-out effect at low temperatures, which is typically observed in doped semiconductors [152]. Considering the embedded MnGe 


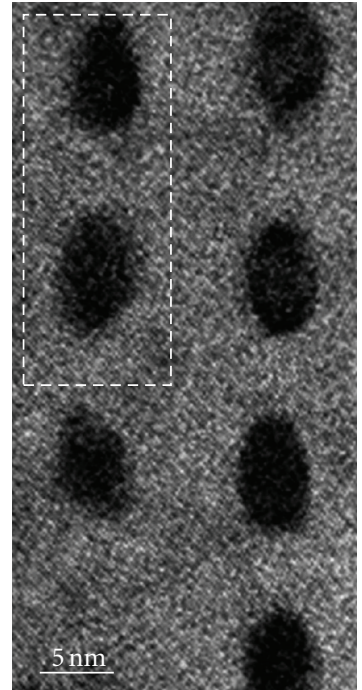

(a)

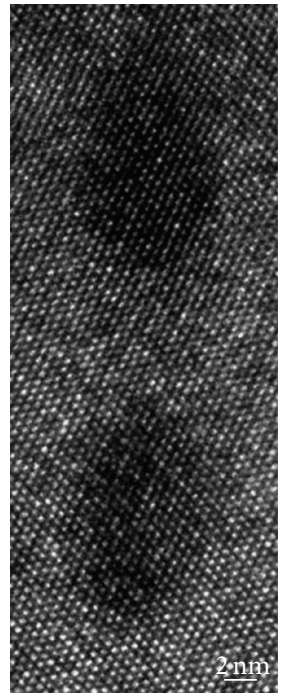

(b)

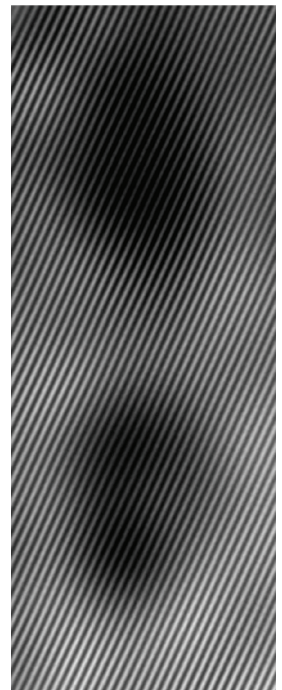

(c)

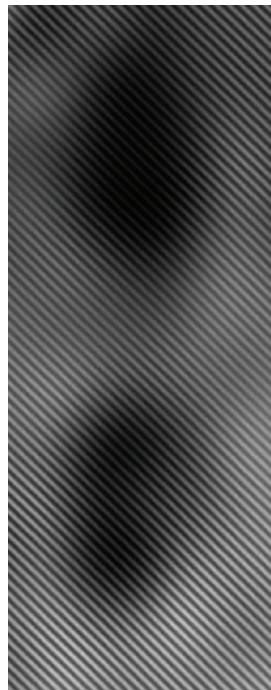

(d)

FIGURE 13: (a) A typical TEM image showing several vertically aligned MnGe nanodots. (b) The HRTEM images of the MnGe nanodots (the selected area in (a)) showing a perfect diamond structure as the Ge matrix. (c, d) Bragg filtering of $\pm(111)(\mathrm{c})$ and $\pm(1 \overline{11})(\mathrm{d})$ reflections, respectively, where no dislocation or distortion was observed. The dark contrast of the nanodots indicates the existence of significant strain.

nanodots, the rise in resistivities at low temperatures also suggests a strong localization of carriers, which takes place at the $\mathrm{Mn}$ sites and/or at the $\mathrm{MnGe/Ge}$ interfaces, similar to the scenario of MnSb clusters in InMnSb crystals [153]. The temperature-dependent resistivity can be generally described by $[154]$

$$
\rho(T)=\rho_{0} \exp \left[\left(\frac{T_{0}}{T}\right)^{1 / \alpha}\right]
$$

where $\rho(T)$ is the temperature-dependent resistivity; $\rho_{0}$ and $T_{0}$ denote material parameters, $\alpha$ is a dimensionality parameter: $\alpha=2$. For one-dimensional (1D), $\alpha=3$ for $2-d$, and $\alpha=4$ for $3-d$ systems. In order to reveal the carrier transport mechanisms at different temperature regions, fittings were performed in the plots of $\ln \rho$ as a function of $T^{-\alpha}$ (Figure 14(a)). The best fittings were found when $\alpha$ equals 1 and 4 in the high-temperature and low-temperature regions, respectively, corresponding to the carrier transport via the band conduction [155] (thermal activation of acceptors) and the 3-d Mott's variable range hopping processes [154]. According to the fitting results to (2), the obtained nanodot arrays show a dominated hopping process below $10 \mathrm{~K}$. At such a low temperature, the majority of free holes are recaptured by the acceptors. As a result, the free-hole band conduction becomes less important and hole hopping directly between acceptors in the impurity band contributes mostly to the conductivity [155]. Above $100 \mathrm{~K}$, the conduction is dominated by the thermal activation of the holes (the band conduction). A thermal activation energy $\left(E_{\alpha}\right)$ of $15 \mathrm{meV}$ can be obtained from (2) with $\alpha=1$ and $E_{\alpha}=T_{0} K_{B}$ where $K_{B}$ is the Boltzmann constant. This activation energy does not correspond to any known acceptor energy levels due to Mn doping in Ge, consistent with results shown in [156].
To explore practical applications for our extraordinary nanodot arrays, the MR measurements were performed from 2 to $300 \mathrm{~K}$ with an external magnetic field up to 10 Tesla. Figure 14(b) shows the plots of temperature-dependent MR at given magnetic fields (5 and 10 Tesla) for the nanodot arrays. Under a strong magnetic field, the MR in the region of variable range-hopping conduction can be described by $[157,158]$

$$
\operatorname{MR}(H)=\exp \left[\frac{c}{\left(\lambda^{2} T\right)^{1 / 3}}\right]-1
$$

where the magnetic length $\lambda$ equals $(c \hbar / e H)^{1 / 2}$ and $\mathrm{C}$ is a field- and temperature-independent constant. Note that (3) is only valid in a strong-field limit [157-159]. The inset in Figure 14(b) shows the best fitting results, in which a linear behavior of MR versus $T^{-1 / 3}$ is obtained, further confirming the hopping conduction mechanisms $(T \leq 8 \mathrm{~K})$. Note that the absolute values of MRs were used for the fitting purpose. These fitting results are reasonably close to the obtained hopping regions determined from the zero-magnetic-field resistivity measurements ( $T \leq 10 \mathrm{~K}$, Figure $14(\mathrm{a})$ ).

It is striking to observe that the coherent MnGe nanodot arrays present a large and positive MR up to $900 \%$ at $2 \mathrm{~K}$ (Figure 14(c)). Traditionally, the positive MR is attributed to the Lorentz force in the semiconductor matrix, which deflects the carriers during the transport process [159]. The resulting MR is positive and proportional to $(\mu H)^{2}$ under low magnetic fields ( $H \leq 1$ Tesla in our case) where $\mu$ is the semiconductor mobility (units $\mathrm{m}^{2} \mathrm{~V}^{-1} \mathrm{~S}^{-1}$ or $\mathrm{T}^{-1}$ ) and $H$ is the magnetic field. However, with a simple calculation, the estimated orbital MR is too small to explain the large MR observed from the nanodot arrays. Instead, we anticipate that, besides the effect of orbital MR, the high-density 

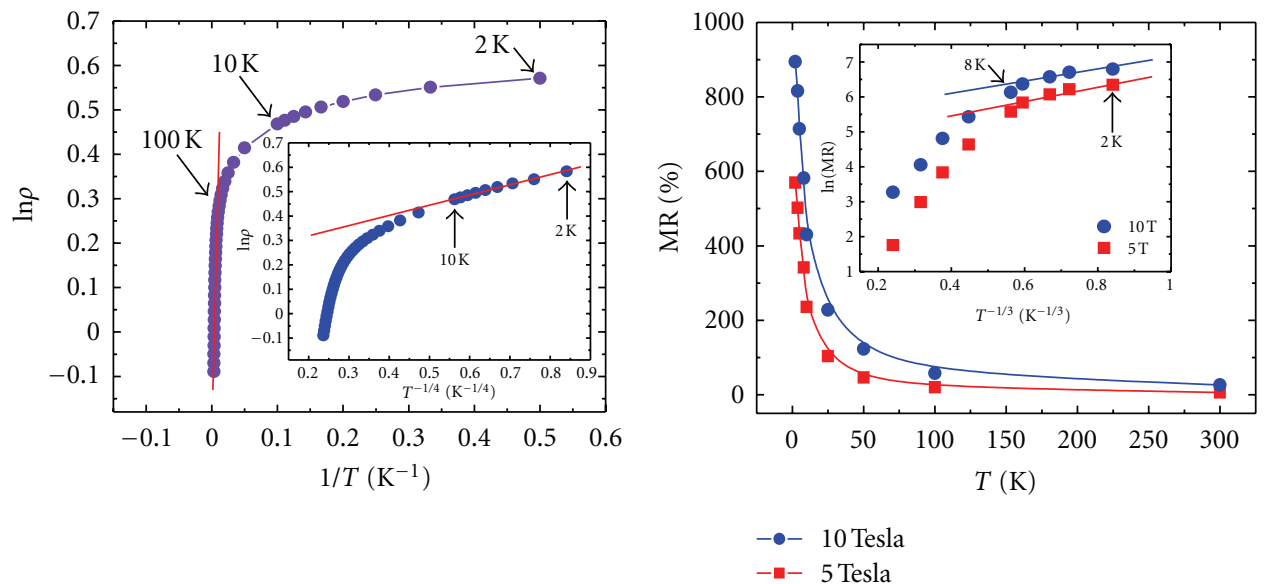

(a)

(b)

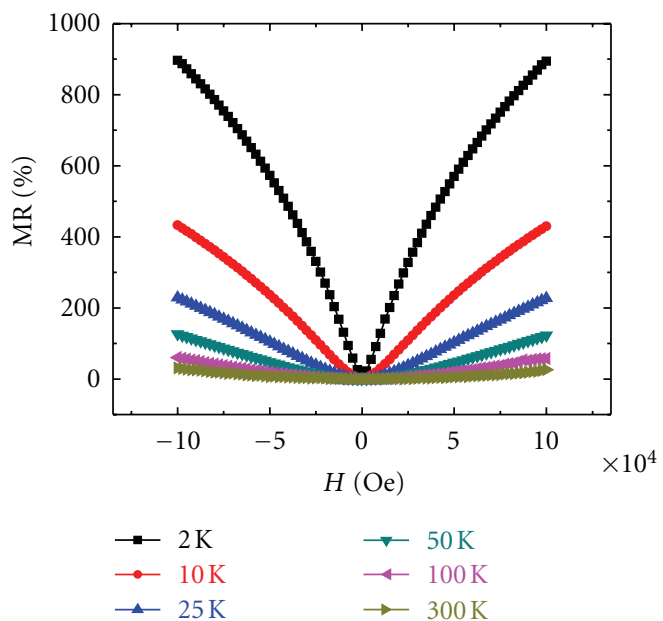

(c)

FIgURE 14: Magnetotransport measurements for the MnGe nanodot arrays. (a) The temperature-dependent resistivity (ln $\rho$ versus $T^{-1}$ ) and the inset displays the plot of $\ln \rho$ versus $T^{-1 / 4}$. (b) Temperature-dependent MR under fixed magnetic fields of 5 and 10 Tesla and the inset showing the plot of $\ln (\mathrm{MR})$ versus $T^{-1 / 3}$. (c) Positive MRs at different temperatures and different magnetic fields.

magnetic nanodots could significantly contribute to the large MR ratios due to an enhanced geometric MR effect, from which the current path may be significantly deflected when external magnetic fields were applied to the magnetic nanostructures $[57,160]$. To elucidate the underlying physics of the geometrical effect, we consider a thin Hall bar geometry with a measurement current applied in the $x$-direction, a Hall voltage in the $y$ direction, $z$ direction normal to the sample surface, and an external magnetic field $H$ parallel to $z$. For semiconductors, the current density and the total electric field can be described by $j=\bar{\sigma} E$, where the magnetoconductivity tensor is given by $[57,160]$

$$
(\bar{\sigma} H)=\left(\begin{array}{ccc}
\frac{\sigma}{1+\beta^{2}} & \frac{\sigma \beta^{2}}{1+\beta^{2}} & 0 \\
\frac{-\sigma \beta^{2}}{1+\beta^{2}} & \frac{\sigma}{1+\beta^{2}} & 0 \\
0 & 0 & \sigma
\end{array}\right) .
$$

Here, $\beta=\mu H$. At zero magnetic field, $\beta$ vanishes. The conductivity tensor is diagonal when lacking of the magnetic field; and the current density can be simply described by $j=\sigma E$. Since the electric field is normal to the surface of a metallic inclusion and $j \| \sigma E$, the current flowing through the material is concentrated into the metallic region which behaves like a "short circuit" [35]. As a result, the inclusion of metallic clusters can lead to a higher conduction than that of a homogeneous semiconductor $[57,160]$. However, at high magnetic fields $(\beta>>1)$, the off-diagonal terms of $\bar{\sigma}(H)$ dominate. Equivalently, the Hall angle between $j$ and $E$ approaches $90^{\circ}(j \perp E)$; and the current becomes tangent to the nanodots. This further indicates that the current is deflected to flow around the nanodots, resembling an "open circuit" state [35]. The transition from the "short circuit" at the zero field to the "open circuit" at high fields produces an increase of resistance, that is, a positive geometrically enhanced MR [160]. 
5.4. Discussions. The introduction of high percentage $\mathrm{Mn}$ $(x \sim 20 \%)$ generates metallic behavior in Mn-doped Ge. Such heavily doped samples possess nanoscale MnGe dots. With a superlattice approach, we have successfully fabricated extraordinarily coherent and self-organized MnGe nanodot arrays embedded in the Ge and GaAs matrixes by low temperature MBE. A high yield of such aligned nanodot arrays was confirmed on different substrates, showing an ideal controllability and reproducibility. More importantly, giant positive magnetoresistances were obtained due to the geometrically enhanced effect. We anticipate that these studies will advance the development of MnGe magnetic semiconductors and/or other similar systems. The obtained coherent and self-assembled nanostructures could be potentially used as the building blocks in the high-density magnetic memories, sensors, MTJs, and other spintronic devices, enabling a new generation of low dissipation magnetoelectronic devices.

\section{Summary and Prospective}

This paper is a review of theoretical and experimental progress that has been achieved in understanding ferromagnetism and related electronic properties in the $\mathrm{Mn}_{x} \mathrm{Ge}_{1-x}$ DMSs, and MnGe metallic nanodots. Interest in DMS ferromagnetism is motivated by the possibility to engineer systems that combine many of the technologically useful features of ferromagnetic and semiconducting materials. This goal has been achieved to an impressive degree in (III, $\mathrm{Mn}) \mathrm{V}$ DMSs, and further progress can be anticipated in the future. However, due to the low $T_{c}$ of (III, Mn)V DMSs, the spintronics research seemly reaches a critical bottleneck, where achieving a high $T_{c}$ DMS becomes an intriguing and challenging task. Fortunately, the $\mathrm{Mn}_{x} \mathrm{Ge}_{1-x}$ material system offers a possible route towards higher $T_{c}$. The structural and magnetic properties can be adjusted simply by modifying the system dimensions from 3- $d$ thin films to $0 \mathrm{D}$ quantum dots. A high $T_{c}$ in excess of $400 \mathrm{~K}$ can be obtained and is presumably attributed to the quantum confinement effect, which strengthens the hybridation between the localized $\mathrm{Mn}$ impurities and iterant holes. Bound magnetic polarons may also exist since this system falls into a regime where $\mathrm{Mn}$ concentrations are much larger than that of holes. Nevertheless, the high $T_{c}$ in the low-dimensional system suggests that the quantum structure exibits extraordinary properties that significantly differ from these of bulk films. The future progress would rely on the precise theoretical understanding of the quantum confinement effect on ferromagnetism.

It is well known that in order to achieve functioning spintronic devices working at ambient temperatures, it requires the following criteria: (i) the ferromagnetic transition temperature should safely exceed room temperature, (ii) the mobile charge carriers should respond strongly to changes in the ordered magnetic state, and (iii) the material should retain fundamental semiconductor characteristics, including sensitivity to doping and light and electric fields produced by gate charges. For more than a decade, these three key issues have been the focus of intense experimental and theoretical research. Progress has been also made in achieving field-controlled ferromagnetism in (III, Mn)As system, even though the controllability remains at low temperatures because of low $T_{c}$. Therefore, the critical challenge now is either to continue increasing $T_{c}$ in (III, Mn)As, or to look for a new DMS system with both high Curie temperature $\left(T_{c}>>300 \mathrm{~K}\right)$ and the field-controlled ferromagnetism to satisfy all these three criteria. The experimental results of field-controlled ferromagnetism in the $\mathrm{Mn}_{0.05} \mathrm{Ge}_{0.95}$ QDs suggest that the ferromagnetism in this system sensitively responds to the electrical field via the hole-mediated effect, similar to that in (III, Mn)As system. Therefore, with a much higher $T_{c}$ compared with III-V DMS, the $\mathrm{Mn}_{x} \mathrm{Ge}_{1-x}$ nanostructures could become one of the most promising candidates to achieve room-temperature operation.

On the other hand, the metallic MnGe nanodots with a high percentage of $\mathrm{Mn}(\sim 20 \%)$ show perfect lattice coherence with the Ge matrix. Such a ferrromagnet/semiconductor hybrid system is extremely useful owing to their high-quality defect-free interface, where a high efficient spin injection can be realized without a need of oxides such as $\mathrm{MgO}$ (spin filter). We also hope to encourage more research efforts in this direction because of the excellent compatibility of MnGe nanodots with the current CMOS technology.

\section{Acknowledgments}

F. Xiu would like to acknowledge startup funds from the Department of Electrical and Computer Engineering, Colleage of Engineering, and Microelectronics Research Center (MRC) at Iowa State University. F. Xiu thanks Professor Kang L. Wang (UCLA, USA), Professor Jin Zou (Queensland University, Australia), and Professor Yong Wang (Zhejiang University, China) for their contributions to this work.

\section{References}

[1] J. S. Kulkarni, O. Kazakova, D. Erts, M. A. Morris, M. T. Shaw, and J. D. Holmes, "Structural and magnetic characterization of $\mathrm{Ge}_{0.99} \mathrm{Mn}_{0.01}$ nanowire arrays," Chemistry of Materials, vol. 17, no. 14, pp. 3615-3619, 2005.

[2] H. Ohno, D. Chiba, F. Matsukura et al., "Electric-field control of ferromagnetism," Nature, vol. 408, no. 6815, pp. 944-946, 2000.

[3] K. Brunner, "Si/Ge nanostructures," Reports on Progress in Physics, vol. 65, no. 1, pp. 27-72, 2002.

[4] T. C. Schulthess and W. H. Butler, "Electronic structure and magnetic interactions in Mn doped semiconductors," Journal of Applied Physics, vol. 89, no. 11, pp. 7021-7023, 2001.

[5] M. van Schilfgaarde and O. N. Mryasov, "Anomalous exchange interactions in III-V dilute magnetic semiconductors," Physical Review B, vol. 63, no. 23, Article ID 233205, 2001.

[6] Y. F. Chen, W. N. Lee, J. H. Huang et al., "Growth and magnetic properties of self-assembled (In, Mn)As quantum dots," Journal of Vacuum Science and Technology B, vol. 23, no. 4, pp. 1376-1378, 2005.

[7] E. Arras, F. Lançon, I. Slipukhina et al., "Interface-driven phase separation in multifunctional materials: The case of the ferromagnetic semiconductor GeMn," Physical Review B, vol. 85, no. 11, 10 pages, 2012. 
[8] C. Liu, F. Yun, and H. Morkoç, "Ferromagnetism of $\mathrm{ZnO}$ and GaN: a review," Journal of Materials Science, vol. 16, no. 9, pp. 555-597, 2005.

[9] M. Bolduc, C. Awo-Affouda, A. Stollenwerk et al., "Above room temperature ferromagnetism in $\mathrm{Mn}$-ion implanted $\mathrm{Si}$," Physical Review B, vol. 71, no. 3, Article ID 033302, 4 pages, 2005.

[10] T. Jungwirth, J. Sinova, J. Masek, J. Kucera, and A. H. MacDonald, "Theory of ferromagnetic (III,Mn)V semiconductors," Reviews of Modern Physics, vol. 78, no. 3, pp. 809-864, 2006.

[11] W. Lu and C. M. Lieber, "Semiconductor nanowires," Journal of Physics D, vol. 39, no. 21, article R387, 2006.

[12] P. Lyu and K. Moon, "Ferromagnetism in diluted magnetic semiconductor quantum dot arrays embedded in semiconductors," European Physical Journal B, vol. 36, no. 4, pp. 593598, 2003.

[13] M. I. van der Meulen, N. Petkov, M. A. Morris et al., "Single crystalline $\mathrm{Ge}_{1-x} \mathrm{Mn}_{x}$ nanowires as building blocks for nanoelectronics," Nano Letters, vol. 9, no. 1, pp. 50-56, 2009.

[14] S. Maekawa, Concepts in Spin Electronics, Oxford University Press, 2006.

[15] K. L. Wang, S. G. Thomas, and M. O. Tanner, "SiGe band engineering for MOS, CMOS and quantum effect devices," Journal of Materials Science, vol. 6, no. 5, pp. 311-324, 1995.

[16] R. N. Bhatt, M. Berciu, M. P. Kennett, and X. Wan, "Diluted magnetic semiconductors in the low carrier density regime," Journal of Superconductivity: Incorporating Novel Magnetism, vol. 15, no. 1, pp. 71-83, 2002.

[17] Y. J. Cho, C. H. Kim, H. S. Kim et al., "Ferromagnetic $\mathrm{Ge}_{1-x} \mathrm{M}_{x}(\mathrm{M}=\mathrm{Mn}, \mathrm{Fe}$, and $\mathrm{Co})$ nanowires," Chemistry of Materials, vol. 20, no. 14, pp. 4694-4702, 2008.

[18] M. Berciu and R. N. Bhatt, "Effects of disorder on ferromagnetism in diluted magnetic semiconductors," Physical Review Letters, vol. 87, no. 10, Article ID 107203, 4 pages, 2001.

[19] L. J. Lauhon, M. S. Gudlksen, D. Wang, and C. M. Lieber, "Epitaxial core-shell and core-multishell nanowire heterostructures," Nature, vol. 420, no. 6911, pp. 57-61, 2002.

[20] N. Nepal, M. O. Luen, J. M. Zavada, S. M. Bedair, P. Frajtag, and N. A. El-Masry, "Electric field control of room temperature ferromagnetism in III-N dilute magnetic semiconductor films," Applied Physics Letters, vol. 94, no. 13, Article ID 132505, 3 pages, 2009.

[21] T. Kanki, H. Tanaka, and T. Kawai, "Electric control of room temperature ferromagnetism in a $\mathrm{Pb}\left(\mathrm{Zr}_{0.2} \mathrm{Ti}_{0.8}\right) \mathrm{O}_{3} /$ $\mathrm{La}_{0.85} \mathrm{Ba}_{0.15} \mathrm{MnO}_{3}$ field-effect transistor," Applied Physics Letters, vol. 89, no. 24, Article ID 242506, 3 pages, 2006.

[22] J. Philip, A. Punnoose, B. I. Kim et al., "Carrier-controlled ferromagnetism in transparent oxide semiconductors," $\mathrm{Na}$ ture Materials, vol. 5, no. 4, pp. 298-304, 2006.

[23] D. Chiba, F. Matsukura, and H. Ohno, "Electric-field control of ferromagnetism in (Ga,Mn)As," Applied Physics Letters, vol. 89, no. 16, Article ID 162505, 3 pages, 2006.

[24] Y. Rheem, B.-Y. Yoo, W. P. Beyermann, and N. V. Myung, "Magneto-transport studies of single ferromagnetic nanowire," Physica Status Solidi A, vol. 204, no. 12, pp. 4004-4008, 2007.

[25] H.-J. Choi, H. K. Seong, J. Chang et al., "Single-crystalline diluted magnetic semiconductor GaN:Mn nanowires," Advanced Materials, vol. 17, no. 11, pp. 1351-1356, 2005.

[26] T. Miyoshi, T. Matsui, H. Tsuda, H. Mabuchi, and K. Morii, "Magnetic and electric properties of $\mathrm{Mn}_{5} \mathrm{Ge}_{3} / \mathrm{Ge}$ nanostructured films," Journal of Applied Physics, vol. 85, no. 8, Article ID 5372, 3 pages, 1999.
[27] Y. D. Park, A. T. Hanbicki, S. C. Erwin et al., "A group-IV ferromagnetic semiconductor: $\mathrm{Mn}_{x} \mathrm{Ge}_{1-x}$," Science, vol. 295, no. 5555, pp. 651-654, 2002.

[28] F. Tsui, L. He, L. Ma et al., "Novel germanium-based magnetic semiconductors," Physical Review Letters, vol. 91, no. 17, Article ID 177203, 4 pages, 2003.

[29] F. D’Orazio, F. Lucari, N. Pinto, L. Morresi, and R. Murri, "Toward room temperature ferromagnetism of Ge:Mn systems," Journal of Magnetism and Magnetic Materials, vol. 272-276, part 3, pp. 2006-2007, 2004.

[30] O. Kazakova, J. S. Kulkarni, J. D. Holmes, and S. O. Demokritov, "Room-temperature ferromagnetism in $\mathrm{Ge}_{1-x} \mathrm{Mn}_{x}$ nanowires," Physical Review B, vol. 72, no. 9, Article ID 094415, 6 pages, 2005.

[31] A. P. Li, J. F. Wendelken, J. Shen, L. C. Feldman, J. R. Thompson, and H. H. Weitering, "Magnetism in $\mathrm{Mn}_{x} \mathrm{Ge}_{1-x}$ semiconductors mediated by impurity band carriers," Physical Review B, vol. 72, no. 19, Article ID 195205, 9 pages, 2005.

[32] E. Demidov, Y. A. Danilov, V. V. Podol'skiǔ, V. P. Lesnikov, M. V. Sapozhnikov, and A. I. Suchkov, "Ferromagnetism in epitaxial germanium and silicon layers supersaturated with manganese and iron impurities," JETP Letters, vol. 83, no. 12, pp. 568-571, 2006.

[33] M. Jamet, A. Barski, T. Devillers et al., "High-curie-temperature ferromagnetism in self-organized $\mathrm{Ge}_{1-x} \mathrm{Mn}_{x}$ nanocolumns," Nature Materials, vol. 5, no. 8, pp. 653-659, 2006.

[34] B. A. Collins, Y. S. Chu, L. He, Y. Zhong, and F. Tsui, "Dopant stability and strain states in Co and Mn-doped Ge (001) epitaxial films," Physical Review B, vol. 77, no. 19, Article ID 193301, 4 pages, 2008.

[35] M. Ogawa, X. Han, Z. Zhao, Y. Wang, K. L. Wang, and J. Zou, "Mn distribution behaviors and magnetic properties of GeMn films grown on Si (001) substrates," Journal of Crystal Growth, vol. 311, no. 7, pp. 2147-2150, 2009.

[36] R. Tsuchida, J. T. Asubar, Y. Jinbo, and N. Uchitomi, "MBE growth and properties of GeMn thin films on (001) GaAs," Journal of Crystal Growth, vol. 311, no. 3, pp. 937-940, 2009.

[37] S. Cho, S. Choi, S. C. Hong et al., "Ferromagnetism in Mndoped Ge," Physical Review B, vol. 66, no. 3, Article ID 033303, 3 pages, 2002.

[38] S. Pramanik, S. Bandyopadhyay, and M. Cahay, "Spin transport in nanowires," in Proceedings of the 3rd IEEE Nanotechnology Conference (IEEE-NANO '03), vol. 2, pp. 87-90, 2003.

[39] J. Chen, K. L. Wang, and K. Galatsis, "Electrical field control magnetic phase transition in nanostructured $\mathrm{Mn}_{x} \mathrm{Ge}_{1-x}$," Applied Physics Letters, vol. 90, no. 1, Article ID 012501, 3 pages, 2007.

[40] F. Xiu, Y. Wang, J. Kim et al., "Electric-field-controlled ferromagnetism in high-Curie-temperature $\mathrm{Mn}_{0.05} \mathrm{Ge}_{0.95}$ quantum dots," Nature Materials, vol. 9, pp. 337-344, 2010.

[41] S. Maekawa, "Spin-dependent transport in magnetic nanostructures," Journal of Magnetism and Magnetic Materials, vol. 272-276, no. 1, pp. E1459-E1463, 2004.

[42] X. Lou, C. Adelmann, S. A. Crooker et al., "Electrical detection of spin transport in lateral ferromagnet-semiconductor devices," Nature Physics, vol. 3, no. 3, pp. 197-202, 2007.

[43] A. P. Li, C. Zeng, K. van Benthem et al., "Dopant segregation and giant magnetoresistance in manganese-doped germanium," Physical Review B, vol. 75, no. 20, Article ID 201201, 4 pages, 2007.

[44] S. Majumdar, S. Mandal, A. K. Das, and S. K. Ray, "Synthesis and temperature dependent photoluminescence properties of Mn doped Ge nanowires," Journal of Applied Physics, vol. 105, no. 2, Article ID 024302, 5 pages, 2009. 
[45] H.-K. Seong, U. Kim, E. K. Jeon et al., "Magnetic and electrical properties of single-crystalline mn-doped Ge nanowires," The Journal of Physical Chemistry C, vol. 113, no. 25, pp. 10847-10852, 2009.

[46] G. Zhang, K. Tateno, T. Sogawa, and H. Nakano, "Vertically aligned $\mathrm{GaP} / \mathrm{GaAs}$ core-multishell nanowires epitaxially grown on Si substrate," Applied Physics Express, vol. 1, Article ID 064003, 3 pages, 2008.

[47] H.-Y. Wang and M. C. Qian, "Electronic and magnetic properties of $\mathrm{Mn} / \mathrm{Ge}$ digital ferromagnetic heterostructures: an ab initio investigation," Journal of Applied Physics, vol. 99, no. 8, Article ID 08D705, 3 pages, 2006.

[48] Y. Q. Chang, D. B. Wang, X. H. Luo et al., "Synthesis, optical, and magnetic properties of diluted magnetic semiconductor $\mathrm{Zn}_{1-x} \mathrm{Mn}_{x} \mathrm{O}$ nanowires via vapor phase growth," Applied Physics Letters, vol. 83, no. 19, pp. 4020-4022, 2003.

[49] J. B. Cui and U. J. Gibson, "Electrodeposition and room temperature ferromagnetic anisotropy of $\mathrm{Co}$ and $\mathrm{Ni}$-doped ZnO nanowire arrays," Applied Physics Letters, vol. 87, no. 13, Article ID 133108, 3 pages, 2005.

[50] J. M. Baik and J. L. Lee, "Fabrication of vertically well-aligned ( $\mathrm{Zn}, \mathrm{Mn}) \mathrm{O}$ nanorods with room temperature ferromagnetism," Advanced Materials, vol. 17, no. 22, pp. 2745-2748, 2005.

[51] F. J. Brieler, P. Grundmann, M. Fröba et al., "Formation of $\mathrm{Zn}_{1-x} \mathrm{Mn}_{x} \mathrm{~S}$ nanowires within mesoporous silica of different pore sizes," Journal of the American Chemical Society, vol. 126, no. 3, pp. 797-807, 2004.

[52] P. V. Radovanovic, C. J. Barrelet, S. Gradecak, F. Qian, and C. M. Lieber, "General synthesis of manganese-doped II-VI and III-V semiconductor nanowires," Nano Letters, vol. 5, no. 7, pp. 1407-1411, 2005.

[53] M. Rovezzi, T. Devillers, E. Arras, F. d'Acapito, A. Barski, M. Jamet, and P. Pochet. "Atomic structure of Mn-rich nanocolumns probed by X-ray absorption spectroscopy," Applied Physics Letters, vol. 92, no. 24, 3 pages, 2008.

[54] H. C. Jeon, K. J. Chung, K. J. Chung, T. W. Kang, and T. W. Kim, "Enhancement of the ferromagnetic transition temperature in self-assembled $\left(\mathrm{Ga}_{1-x} \mathrm{Mn}_{x}\right)$ As quantum wires," Japanese Journal of Applied Physics, vol. 43, pp. L963-L965, 2004.

[55] Y. Ji, A. Hoffmann, J. S. Jiang, and S. D. Bader, "Spin injection, diffusion, and detection in lateral spin-valves," Applied Physics Letters, vol. 85, no. 25, pp. 6218-6220, 2004.

[56] Y.-J. Zhao, T. Shishidou, and A. J. Freeman, "RudermanKittel-Kasuya-Yosida-like ferromagnetism in $\mathrm{Mn}_{x} \mathrm{Ge}_{1-x}$," Physical Review Letters, vol. 90, no. 4, Article ID 047204, 4 pages, 2003.

[57] S. U. Yuldashev, Y. Shon, Y. H. Kwon et al., "Enhanced positive magnetoresistance effect in GaAs with nanoscale magnetic clusters," Journal of Applied Physics, vol. 90, no. 6, pp. 3004-3006, 2001.

[58] A. Stroppa, S. Picozzi, A. Continenza, and A. J. Freeman, "Electronic structure and ferromagnetism of Mn-doped group-IV semiconductors," Physical Review B, vol. 68, no. 15, Article ID 155203, 9 pages, 2003.

[59] Y. Ji, A. Hoffmann, J. S. Jiang, J. E. Pearson, and S. D. Bader, "Non-local spin injection in lateral spin valves," Journal of Physics D, vol. 40, no. 5, article S13, pp. 1280-1284, 2007.

[60] H. Akai, "Ferromagnetism and its stability in the diluted magnetic semiconductor (In, Mn)As," Physical Review Letters, vol. 81, no. 14, pp. 3002-3005, 1998.

[61] T. Dietl, H. Ohno, and F. Matsukura, "Hole-mediated ferromagnetism in tetrahedrally coordinated semiconductors,"
Physical Review B, vol. 63, no. 19, Article ID 195205, 21 pages, 2001.

[62] S. Picozzi and M. Lezaic, "Ab-initio study of exchange constants and electronic structure in diluted magnetic group-IV semiconductors," New Journal of Physics, vol. 10, Article ID 055017, 2008.

[63] X. Luo, S. B. Zhang, and S.-H. Wei, "Theory of Mn supersaturation in Si and Ge," Physical Review B, vol. 70, no. 3, Article ID 033308, 3 pages, 2004.

[64] S. C. Erwin and A. G. Petukhov, "Self-compensation in manganese-doped ferromagnetic semiconductors," Physical Review Letters, vol. 89, no. 22, Article ID 227201, 4 pages, 2002.

[65] G. A. Prinz, "Magnetoelectronics," Science, vol. 282, no. 5394, pp. 1660-1663, 1998.

[66] F. J. Jedema, H. B. Heersche, A. T. Filip, J. J. A. Baselmans, and B. J. van Wees, "Electrical detection of spin precession in a metallic mesoscopic spin valve," Nature, vol. 416, no. 6882, pp. 713-716, 2002.

[67] H. Chen, W. Zhu, E. Kaxiras, and Z. Zhang, "Optimization of Mn doping in group-IV-based dilute magnetic semiconductors by electronic codopants," Physical Review B, vol. 79, no. 23, Article ID 235202, 13 pages, 2009.

[68] S. O. Valenzuela and M. Tinkham, "Spin-polarized tunneling in room-temperature mesoscopic spin valves," Applied Physics Letters, vol. 85, no. 24, pp. 5914-5916, 2004.

[69] S. Garzon, I. Zutic, and R. A. Webb, "Temperature-dependent asymmetry of the nonlocal spin-injection resistance: evidence for spin nonconserving interface scattering," Physical Review Letters, vol. 94, no. 17, Article ID 176601, 4 pages, 2005.

[70] B. I. Shklovskii and A. L. Efros, Electronics Properties of Doped Semiconductors, Spinger, 1984.

[71] N. Poli, M. Urech, V. Korenivski, and D. B. Haviland, "Spinflip scattering at Al surfaces," Journal of Applied Physics, vol. 99, no. 8, Article ID 08H701, 3 pages, 2006.

[72] M. Peressi, A. Debernardi, S. Picozzi, F. Antoniella, and A. Continenza, "Half-metallic Mn-doped $\mathrm{Si}(x) \mathrm{Ge}(1-x)$ alloys: a first principles study," Computational Materials Science, vol. 33, no. 1-3, pp. 125-131, 2005.

[73] T. Kimura and Y. Otani, "Local domain structure of exchange-coupled NiFe/CoO nanowire probed by nonlocal spin valve measurement," Journal of Applied Physics, vol. 103, no. 8, Article ID 083915, 5 pages, 2008.

[74] M. Johnson and R. H. Silsbee, "Interfacial charge-spin coupling: injection and detection of spin magnetization in metals," Physical Review Letters, vol. 55, no. 17, pp. 17901793, 1985.

[75] S. Patibandla, S. Pramanik, S. Bandyopadhyay, and G. C. Tepper, "Spin relaxation in a germanium nanowire," Journal of Applied Physics, vol. 100, no. 4, Article ID 044303, 5 pages, 2006.

[76] D. Kelly, J.-E. Wegrowe, T.-K. Truong, X. Hoffer, and J.-P. Ansermet, "Spin-polarized current-induced magnetization reversal in single nanowires," Physical Review B, vol. 68, no. 13, Article ID 134425, 13 pages, 2003.

[77] C. Jaeger, C. Bihler, T. Vallaitis et al., "Spin-glass-like behavior of Ge:Mn," Physical Review B, vol. 74, no. 4, Article ID 045330, 10 pages, 2006.

[78] S. Ahlers, D. Bougeard, H. Riedl et al., "Ferromagnetic Ge(Mn) nanostructures," Physica E, vol. 32, no. 1-2, pp. 422425, 2006.

[79] S. Ahlers, D. Bougeard, N. Sircar et al., "Magnetic and structural properties of $\mathrm{Ge}_{x} \mathrm{Mn}_{1-x}$ films: precipitation of intermetallic nanomagnets," Physical Review B, vol. 74, no. 21, Article ID 214411, 8 pages, 2006. 
[80] C. Bihler, C. Jaeger, T. Vallaitis et al., "Structural and magnetic properties of $\mathrm{Mn}_{5} \mathrm{Ge}_{3}$ clusters in a dilute magnetic germanium matrix," Applied Physics Letters, vol. 88, no. 11, Article ID 112506, 3 pages, 2006.

[81] D. Bougeard, S. Ahlers, A. Trampert, N. Sircar, and G. Abstreiter, "Clustering in a precipitate-free GeMn magnetic semiconductor," Physical Review Letters, vol. 97, no. 23, Article ID 237202, 4 pages, 2006.

[82] T. Devillers, M. Jamet, A. Barski et al., "Structure and magnetism of self-organized $\mathrm{Ge}_{1-x} \mathrm{Mn}_{x}$ nanocolumns on Ge(001)," Physical Review B, vol. 76, no. 20, Article ID 205306, 12 pages, 2007.

[83] Y. D. Park, A. Wilson, A. T. Hanbicki et al., "Magnetoresistance of Mn:Ge ferromagnetic nanoclusters in a diluted magnetic semiconductor matrix," Applied Physics Letters, vol. 78, no. 18, pp. 2739-2741, 2001.

[84] Y. Wang, J. Zou, Z. Zhao, X. Han, X. Zhou, and K. L. Wang, "Direct structural evidences of $\mathrm{Mn}_{11} \mathrm{Ge}_{8}$ and $\mathrm{Mn}_{5} \mathrm{Ge}_{2}$ clusters in $\mathrm{Ge}_{0.96} \mathrm{Mn}_{0.04}$ thin films," Applied Physics Letters, vol. 92, no. 10, Article ID 101913, 3 pages, 2008.

[85] N. Pinto, L. Morresi, M. Ficcadenti et al., "Magnetic and electronic transport percolation in epitaxial $\mathrm{Ge}_{1-x} \mathrm{Mn}_{x}$ films," Physical Review B, vol. 72, no. 16, Article ID 165203, 7 pages, 2005.

[86] E. S. Park, D. H. Kim, and W. T. Kim, "Parameter for glass forming ability of ternary alloy systems," Applied Physics Letters, vol. 86, no. 6, Article ID 061907, 3 pages, 2005.

[87] H.-T. Lin, W.-J. Huang, S.-H. Wang, H.-H. Lin, and T.S. Chin, "Carrier-mediated ferromagnetism in p-Si(100) by sequential ion-implantation of B and Mn," Journal of Physics, vol. 20, no. 9, Article ID 095004, 2008.

[88] A. Verna, L. Ottaviano, M. Passacantando et al., "Ferromagnetism in ion implanted amorphous and nanocrystalline $\mathrm{Mn}_{x} \mathrm{Ge}_{1-x}$, Physical Review B, vol. 74 , no. 8, Article ID 085204, 12 pages, 2006.

[89] F. D’Orazio, F. Lucari, M. Passacantando, P. P. S. Santucci, and A. Verna, "Magneto-optical study of Mn ions implanted in Ge," in Proceedings of the IEEE International of Magnetics Conference (INTERMAG Europe '02), 2002.

[90] L. Liu, N. Chena, Z. Yina, F. Yanga, J. Zhoua, and F. Zhang, "Investigation of Mn-implanted n-type Ge," Journal of Crystal Growth, vol. 265, no. 3-4, pp. 466-470, 2004.

[91] L. Lifeng, C. Nuofu, C. Chenlong, L. Yanli, Y. Zhigang, and Y. Fei, "Magnetic properties of Mn-implanted n-type Ge," Journal of Crystal Growth, vol. 273, no. 1-2, pp. 106-110, 2004.

[92] M. Passacantando, L. Ottaviano, F. D’Orazio et al., “Growth of ferromagnetic nanoparticles in a diluted magnetic semiconductor obtained by $\mathrm{Mn}^{+}$implantation on Ge single crystals," Physical Review B, vol. 73, no. 19, Article ID 195207, 5 pages, 2006.

[93] L. Ottaviano, M. Passacantando, A. Verna et al., "Microscopic investigation of the structural and electronic properties of ion implanted Mn-Ge alloys," Physica Status Solidi A, vol. 204, no. 1, pp. 136-144, 2007.

[94] E. Biegger, L. Staheli, M. Fonin, U. Rudiger, and Y. S. Dedkov, "Intrinsic ferromagnetism versus phase segregation in Mndoped Ge," Journal of Applied Physics, vol. 101, no. 10, Article ID 103912, 5 pages, 2007.

[95] S. Majumdar, A. K. Das, and S. K. Ray, "Magnetic semiconducting diode of $\mathrm{p}-\mathrm{Ge}_{1-x} \mathrm{Mn}_{x} / \mathrm{n}$-Ge layers on silicon substrate," Applied Physics Letters, vol. 94, no. 12, Article ID 122505, 3 pages, 2009.
[96] R. Gunnella, L. Morresi, N. Pinto et al., "Magnetization of epitaxial MnGe alloys on Ge(111) substrates," Surface Science, vol. 577, no. 1, pp. 22-30, 2005.

[97] L. Liu, N. Chen, Y. Wang et al., "Growth and properties of magnetron cosputtering grown $\mathrm{Mn}_{x} \mathrm{Ge}_{1-x}$ on $\mathrm{Si}(001)$," Solid State Communications, vol. 137, no. 3, pp. 126-128, 2006.

[98] H. Li, Y. Wu, T. Liu, S. Wang, Z. Guo, and T. Osipowicz, "Magnetic and transport properties of Ge:Mn granular system," Thin Solid Films, vol. 505, no. 1-2, pp. 54-56, 2006.

[99] W. Zhu, H. H. Weitering, E. G. Wang, E. Kaxiras, and Z. Zhang, "Contrasting growth modes of $\mathrm{Mn}$ on $\mathrm{Ge}(100)$ and $\mathrm{Ge}(111)$ surfaces: subsurface segregation versus intermixing," Physical Review Letters, vol. 93, no. 12, Article ID 126102, 4 pages, 2004.

[100] H. Li, Y. Wu, Z. Guo, P. Luo, and S. Wang, "Magnetic and electrical transport properties of $\mathrm{Ge}_{1-x} \mathrm{Mn}_{x}$ thin films," Journal of Applied Physics, vol. 100, no. 10, Article ID 103908, 9 pages, 2006.

[101] F. D’Orazio, F. Lucari, S. Santucci et al., "Magneto-optical properties of epitaxial $\mathrm{Mn}_{x} \mathrm{Ge}_{1-x}$ films," Journal of Magnetism and Magnetic Materials, vol. 262, no. 1, pp. 158-161, 2003.

[102] C. Zeng, Y. Yao, Q. Niu, and H. H. Weitering, "Linear magnetization dependence of the intrinsic anomalous hall effect," Physical Review Letters, vol. 96, no. 3, Article ID 037204, 4 pages, 2006.

[103] H. Liu and P. Reinke, "Formation of manganese nanostructures on the $\mathrm{Si}(100)-(2 \times 1)$ surface," Surface Science, vol. 602, no. 4, pp. 986-992, 2008.

[104] P. Gambardella, L. Claude, S. Rusponi et al., "Surface characterization of $\mathrm{Mn}_{x} \mathrm{Ge}_{1-x}$ and $\mathrm{Cr}_{y} \mathrm{Mn}_{x} \mathrm{Ge}_{1-x-y}$ dilute magnetic semiconductors," Physical Review B, vol. 75, no. 12, Article ID 125211, 7 pages, 2007.

[105] Y. M. Cho, S. S. Yu, Y. E. Ihm et al., "Neutron irradiation effects on polycrystalline $\mathrm{Ge}_{1-x} \mathrm{Mn}_{x}$ thin films grown by MBE," Current Applied Physics, vol. 6, no. 3, pp. 482-485, 2006.

[106] L. Morresi, N. Pinto, M. Ficcadenti, R. Murri, F. D’Orazio, and F. Lucari, "Magnetic and transport polaron percolation in diluted GeMn films," Materials Science and Engineering B, vol. 126, no. 2-3, pp. 197-201, 2006.

[107] S. Sugahara, K. L. Lee, S. Yada, and M. Tanaka, "Precipitation of amorphous ferromagnetic semiconductor phase in epitaxially grown Mn-doped Ge thin films," Japanese Journal of Applied Physics, vol. 44, no. 46-49, pp. L1426-L1429, 2005.

[108] Y. Wang, J. Zou, Z. Zhao, X. Han, X. Zhou, and K. L. Wang, "Mn behavior in $\mathrm{Ge}_{0.96} \mathrm{Mn}_{0.04}$ magnetic thin films grown on Si," Journal of Applied Physics, vol. 103, no. 6, Article ID 066104, 3 pages, 2008.

[109] M. Passacantando, L. Ottaviano, V. Grossi et al., "Magnetic response of Mn-doped amorphous porous Ge fabricated by ion-implantation," Nuclear Instruments and Methods in Physics Research B, vol. 257, no. 1-2, pp. 365-368, 2007.

[110] Y. X. Chen, S. S. Yan, Y. Fang et al., "Magnetic and transport properties of homogeneous $\mathrm{Mn}_{x} \mathrm{Ge}_{1-x}$ ferromagnetic semiconductor with high Mn concentration," Applied Physics Letters, vol. 90, no. 5, Article ID 052508, 3 pages, 2007.

[111] A. Verna, F. D’Orazio, L. Ottaviano et al., "Magneto-optical investigation of high temperature ion implanted $\mathrm{Mn}_{x} \mathrm{Ge}_{1-x}$ alloy: evidence for multiple contributions to the magnetic response," Physica Status Solidi A, vol. 204, no. 1, pp. 145$151,2007$.

[112] N. Pinto, L. Morresi, R. Gunnella et al., "Growth and magnetic properties of MnGe films for spintronic application," Journal of Materials Science, vol. 14, no. 5-7, pp. 337-340, 2003. 
[113] S. S. Yu, T. T. L. Anh, Y. E. Ihm et al., "Magneto-transport properties of amorphous $\mathrm{Ge}_{1-x} \mathrm{Mn}_{x}$ thin films," Current Applied Physics, vol. 6, no. 3, pp. 545-548, 2006.

[114] S. Yada, S. Sugahara, and M. Tanaka, "Magneto-optical and magnetotransport properties of amorphous ferromagnetic semiconductor $\mathrm{Ge}_{1-x} \mathrm{Mn}_{x}$ thin films," Applied Physics Letters, vol. 93, no. 19, Article ID 193108, 3 pages, 2008.

[115] P. Gambardella, H. Brune, S. S. Dhesi et al., "Paramagnetic Mn impurities on Ge and GaAs surfaces," Physical Review B, vol. 72, no. 4, Article ID 045337, 5 pages, 2005.

[116] F. Tsui, B. A. Collins, L. He et al., "Combinatorial synthesis and characterization of a ternary epitaxial film of Co and $\mathrm{Mn}$ doped Ge (001)," Applied Surface Science, vol. 254, no. 3, pp. 709-713, 2007.

[117] A. Paul and B. Sanyal, "Chemical and magnetic interactions in $\mathrm{Mn}$-and Fe-codoped Ge diluted magnetic semiconductors," Physical Review B, vol. 79, no. 21, Article ID 214438, 5 pages, 2009.

[118] R. R. Gareev, Y. V. Bugoslavsky, R. Schreiber, A. Paul, M. Sperl, and M. Döppe, "Carrier-induced ferromagnetism in $\mathrm{Ge}(\mathrm{Mn}, \mathrm{Fe})$ magnetic semiconductor thin-film structures," Applied Physics Letters, vol. 88, no. 22, Article ID 222508, 3 pages, 2006.

[119] T. Fukushima, K. Sato, H. Katayama-Yoshida, and P. H. Dederichs, "Spinodal decomposition under layer by layer growth condition and high curie temperature quasi-onedimensional nano-structure in dilute magnetic semiconductors," Japanese Journal of Applied Physics, vol. 45, no. 12-16, pp. L416-L418, 2006.

[120] M. Holub, S. Chakrabarti, S. Fathpour, P. Bhattacharya, Y. Lei, and S. Ghosh, "Mn-doped InAs self-organized diluted magnetic quantum-dot layers with Curie temperatures above 300 K," Applied Physics Letters, vol. 85, no. 6, pp. 973-975, 2004.

[121] Y. H. Zheng, J. H. Zhao, J. F. Bi et al., "Cr-Doped InAs selforganized diluted magnetic quantum dots with room-temperature ferromagnetism," Chinese Physics Letters, vol. 24, no. 7, article 092, pp. 2118-2121, 2007.

[122] A. Arrott, "Criterion for ferromagnetism from observations of magnetic isotherms," Physical Review, vol. 108, no. 6, pp. 1394-1396, 1957.

[123] S. M. Sze, Physics of Semiconductor Devices, John Wiley \& Sons, New York, NY, USA, 3rd edition, 2007.

[124] I. V. Ovchinnikov and K. L. Wang, "Voltage sensitivity of Curie temperature in ultrathin metallic films," Physical Review B, vol. 80, no. 1, Article ID 012405, 4 pages, 2009.

[125] T. Dietl and J. Spalek, "Effect of thermodynamic fluctuations of magnetization on the bound magnetic polaron in dilute magnetic semiconductors," Physical Review B, vol. 28, no. 3, pp. 1548-1563, 1983.

[126] D. Kitchen, A. Richardella, P. Roushan, J.-M. Tang, M. E. Flatt, and A. Yazdani, "Hole-mediated interactions of Mn acceptors on GaAs (110) (invited)," Journal of Applied Physics, vol. 101, no. 9, Article ID 09G515, 6 pages, 2007.

[127] S. W. Lim, M. C. Jeong, M. H. Ham, and J. M. Myoung, "Hole-mediated ferromagnetic properties in $\mathrm{Zn}_{1-x} \mathrm{Mn}_{x} \mathrm{O}$ Thin Films," Japanese Journal of Applied Physics, vol. 43, no. 2B, pp. L280-L283, 2004.

[128] H. C. Jeon, Y. S. Jeong, T. W. Kang et al., “ $\left(\operatorname{In}_{1-x} M n_{x}\right)$ As diluted magnetic semiconductor quantum dots with above room temperature ferromagnetic transition," Advanced Materials, vol. 14, no. 23, pp. 1725-1728, 2002.

[129] D. D. Awschalom, D. Loss, and N. Samarth, Eds., Semiconductor Spintronics and Quantum Computation, Springer, New York, NY, USA, 2002.
[130] D. Chiba, F. Matsukura, and H. Ohno, "Electrical magnetization reversal in ferromagnetic III-V semiconductors," Journal of Physics D, vol. 39, no. 13, article R01, pp. R215-R225, 2006.

[131] S. Datta and B. Das, "Electronic analog of the electro-optic modulator," Applied Physics Letters, vol. 56, no. 7, pp. 665667, 1990.

[132] I. Appelbaum and D. J. Monsma, "Transit-time spin fieldeffect transistor," Applied Physics Letters, vol. 90, no. 26, Article ID 262501, 2007.

[133] A. M. Nazmul, S. Kobayashi, S. Sugahara, and M. Tanaka, "Electrical and optical control of ferromagnetism in III$\mathrm{V}$ semiconductor heterostructures at high temperature ( $100 \mathrm{~K})$," Japanese Journal of Applied Physics, vol. 43, no. 2A, pp. L233-L236, 2004.

[134] H. C. Koo, J. H. Kwon, J. Eom, J. Chang, S. H. Han, and M. Johnson, "Control of spin precession in a spin-injected field effect transistor," Science, vol. 325, no. 5947, pp. 1515-1518, 2009.

[135] D. Chiba, M. Sawicki, Y. Nishitani, Y. Nakatani, F. Matsukura, and H. Ohno, "Magnetization vector manipulation by electric fields," Nature, vol. 455, no. 7212, pp. 515-518, 2008.

[136] H. Boukari, P. Kossacki, M. Bertolini et al., "Light and electric field control of ferromagnetism in magnetic quantum structures," Physical Review Letters, vol. 88, no. 20, Article ID 207204, 4 pages, 2002.

[137] T. Dietl, H. Ohno, F. Matsukura, J. Cibert, and D. Ferrand, "Zener model description of ferromagnetism in zinc-blende magnetic semiconductors," Science, vol. 287, no. 5455, pp. 1019-1022, 2000.

[138] F. Matsukura, H. Ohno, A. Shen, and Y. Sugawara, "Transport properties and origin of ferromagnetism in (Ga,Mn)As," Physical Review B, vol. 57, no. 4, pp. R2037-R2040, 1998.

[139] M. Yagi, K.-I. Noba, and Y. Kayanuma, "Self-consistent theory for ferromagnetism induced by photo-excited carriers," Journal of Luminescence, vol. 94-95, pp. 523-527, 2001.

[140] T. Dietl and H. Ohno, "Engineering magnetism in semiconductors," Materials Today, vol. 9, no. 11, pp. 18-26, 2006.

[141] M. Weisheit, S. Fähler, A. Marty, Y. Souche, C. Poinsignon, and D. Givord, "Electric field-induced modification of magnetism in thin-film ferromagnets," Science, vol. 315, no. 5810, pp. 349-351, 2007.

[142] M. Tanaka, "Ferromagnet (MnAs)/III-V semiconductor hybrid structures," Semiconductor Science and Technology, vol. 17, no. 4, pp. 327-341, 2002.

[143] Y. Wang, F. Xiu, Y. Wang et al., "Coherent magnetic semiconductor nanodot arrays," Nanoscale Research Letters, vol. 6, no. 1, 2011.

[144] T. Dietl, "From our readers: self-organized growth controlled by charge states of magnetic impurities," Nature Materials, vol. 5, no. 9, p. 673, 2006.

[145] S. Kuroda, N. Nishizawa, K. Takita et al., "Origin and control of high-temperature ferromagnetism in semiconductors," Nature Materials, vol. 6, no. 6, pp. 440-446, 2007.

[146] G. Kioseoglou, A. T. Hanbicki, J. M. Sullivan et al., "Electrical spin injection from an n-type ferromagnetic semiconductor into a III-V device heterostructure," Nature Materials, vol. 3, no. 11, pp. 799-803, 2004.

[147] T. Dietl, D. D. Awschalom, M. Kaminska, and H. Ohno, Spintronics, Elsevier, 2008.

[148] J. C. Slater, "Atomic radii in crystals," The Journal of Chemical Physics, vol. 41, no. 10, article 3199, 6 pages, 1964.

[149] Q. H. Xie, A. Madhukar, P. Chen, and N. P. Kobayashi, "Vertically self-organized InAs quantum box islands on 
GaAs(100)," Physical Review Letters, vol. 75, no. 13, pp. 25422545, 1995.

[150] G. S. Solomon, J. A. Trezza, A. F. Marshall, and J. S. Harris, "Vertically aligned and electronically coupled growth induced InAs islands in GaAs," Physical Review Letters, vol. 76, no. 6, pp. 952-955, 1996.

[151] X. Z. Liao, J. Zou, D. J. H. Cockayne et al., "Annealing effects on the microstructure of $\mathrm{Ge} / \mathrm{Si}(001)$ quantum dots," Applied Physics Letters, vol. 79, no. 9, pp. 1258-1260, 2001.

[152] S. M. Sze, Physics of Semiconductor Devices, John Wiley \& Sons, New York, NY, USA, 2nd edition, 1981.

[153] Y. Ohno, D. K. Young, B. Beschoten, F. Matsukura, H. Ohno, and D. D. Awschalom, "Electrical spin injection in a ferromagnetic semiconductor heterostructure," Nature, vol. 402, no. 6763, pp. 790-792, 1999.

[154] Y. J. Ma, Z. Zhang, F. Zhou, L. Lu, A. Jin, and C. Gu, "Hopping conduction in single $\mathrm{ZnO}$ nanowires," Nanotechnology, vol. 16, no. 6, pp. 746-749, 2005.

[155] J. P. Han, M. R. Shen, W. W. Cao, A. M. R. Senos, and P. Q. Mantas, "Hopping conduction in Mn-doped ZnO," Applied Physics Letters, vol. 82, no. 1, pp. 67-69, 2003.

[156] N. Pinto, L. Morresi, M. Ficcadenti et al., "Magnetic and electronic transport percolation in epitaxial $\mathrm{Ge}_{1-x} \mathrm{Mn}_{x}$ films," Physical Review B, vol. 72, no. 16, Article ID 165203, 7 pages, 2005.

[157] B. I. Schlovskii and A. L. Efros, Electronics Properties of Doped Semiconductors, Spinger, Berlin, Germany, 1984.

[158] H. Bottger and V. Bryksin, Hopping Conduction in Solids, Akademie, Berlin, Germany, 1985.

[159] K. Ganesan and H. L. Bhat, "Growth, magnetotransport, and magnetic properties of ferromagnetic (In,Mn)Sb crystals," Journal of Applied Physics, vol. 103, no. 4, Article ID 043701, 6 pages, 2008.

[160] S. A. Solin, T. Thio, D. R. Hines, and J. J. Heremans, "Enhanced room-temperature geometric magnetoresistance in inhomogeneous narrow-gap semiconductors," Science, vol. 289, no. 5484, pp. 1530-1532, 2000. 

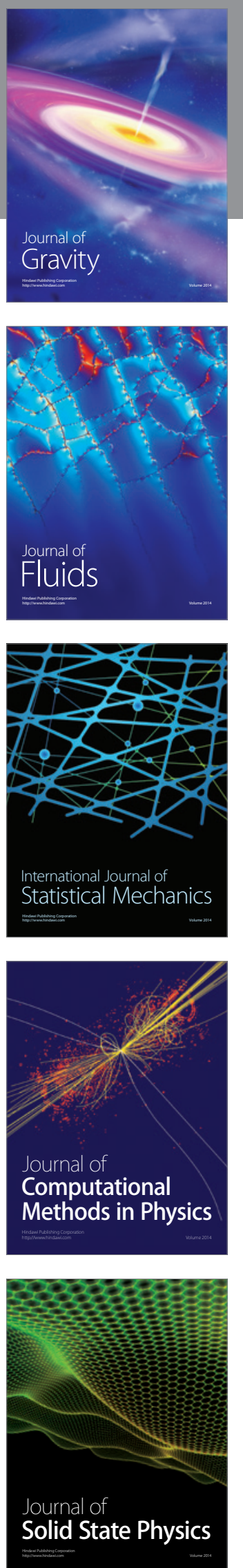
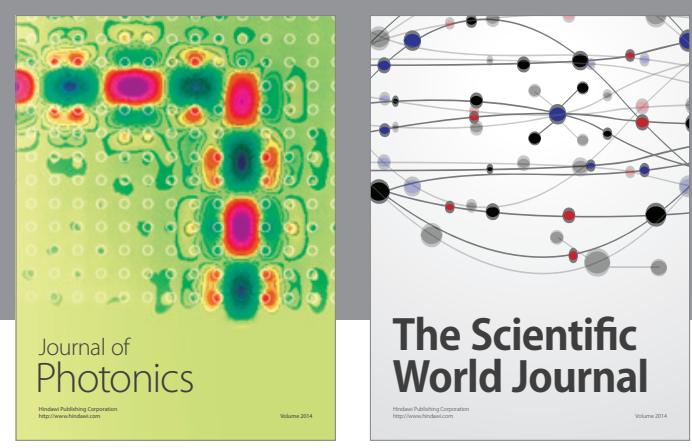

The Scientific World Journal

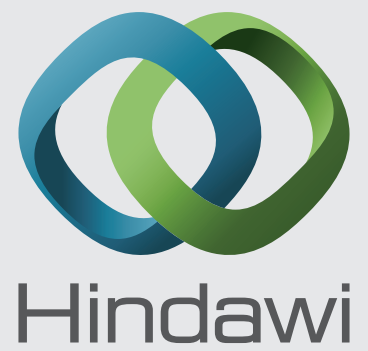

Submit your manuscripts at http://www.hindawi.com
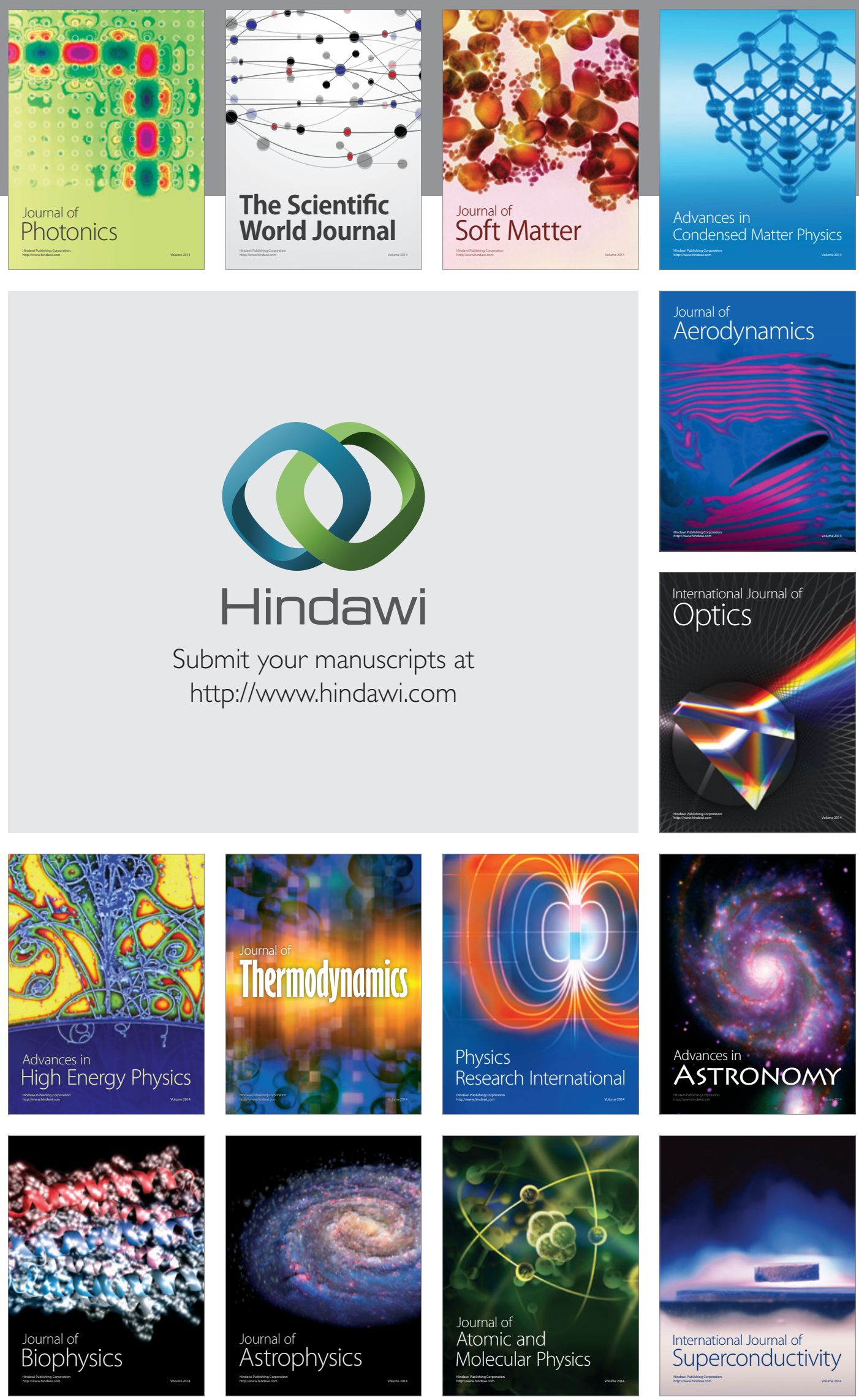
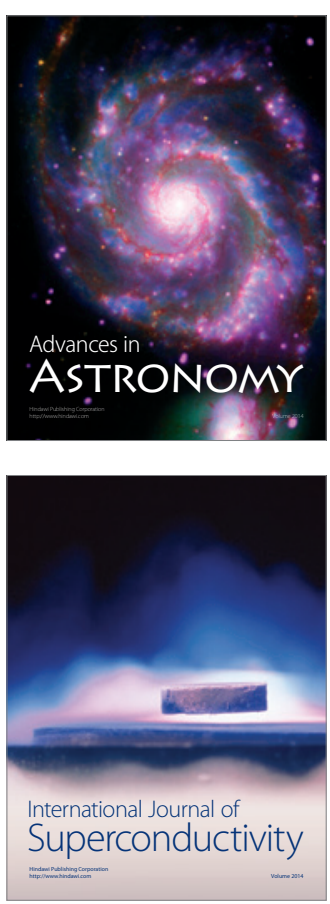